\title{
ON THE OBSERVABILITIES OF THE CHIMNEY MODEL OF THE INTERSTELLAR MEDIUM
}

\author{
FAN Li AND SATORU IKeUChI \\ National Astronomical Observatory, Mitaka, Tokyo \\ Received 1989 August 24; accepted 1989 November 11
}

\begin{abstract}
The Monte Carlo simulation is employed to study the distribution of superbubbles and supernova remnants according to the chimney model of the interstellar medium, taking into account the global spiral pattern in the Galaxy. The observabilities of superbubbles and hot gas within them by $\mathrm{H}$ $21 \mathrm{~cm}$ and diffuse X-ray emission are examined, and we show expected observational characteristics of $\mathrm{H}$ i supershells and an X-ray ridge. These are very similar to what have been observed in our Galaxy. In other words, the structure of interstellar medium with many superbubbles concentrated in spiral arms, the so-called chimney model, does offer a possible explanation to the various observed structures of the interstellar medium.
\end{abstract}

Subject headings: interstellar: matter — nebulae: supernova remnants — radio sources: $21 \mathrm{~cm}$ radiation

\section{INTRODUCTION}

Many observational results have been accumulated during last decade which cannot be easily understood by the standard picture of the interstellar medium (ISM) represented by the three-phase model of McKee and Ostriker (1977).

In addition to the coexistence of giant molecular clouds, $\mathrm{OB}$ associations, $\mathrm{H}$ II regions, and $\mathrm{H}$ I loops, large-scale structures such as supershells (Heiles 1979, 1984) and X-ray ridge from hot gas with temperature higher than $10^{7} \mathrm{~K}$ (Warwick et al. 1985; Koyama et al. 1986) are confirmed in our Galaxy. In order to include these new structures, a new model of ISM called the chimney model has been proposed (Ikeuchi 1987, 1988; Norman and Ikeuchi 1989) as follows. OB stars are formed sequentially from molecular clouds and end with Type II supernova explosions which, unlike Type I supernovae, are bunched in space and time. As a sequence of these explosions, many giant energetic superbubbles (SBs) surrounded by $\mathrm{H}$ I supershells are formed. They break through the Galactic disk and eject energy and mass into the halo. After sufficient cooling, the ejected mass returns to the disk as infalling clouds. Since most giant molecular clouds and $\mathrm{H}$ II regions are associated in the spiral arms, we consider the SBs be formed in the arm regions.

In Paper I (Li and Ikeuchi 1989) we studied the interchange processes of the ISM regulated by supernova remnants (SNRs) and SBs, and the mass circulation effects between the Galactic disk and halo were examined. Although the three-component model by McKee and Ostriker (1977) is basically correct at least in the global structure of solar neighborhood, the evolution of the gaseous halo is much affected by the mass and energy input by SBs.

In this paper, we study the observational consequences of chimney model, especially, in the large-scale structures formed by SBs in $\mathrm{H}_{\mathrm{I}} 21 \mathrm{~cm}$ and diffuse X-ray emissions, and compare them with current observational data.

In $\S$ II, we present the model for our Galaxy and the methods of calculation. In $\S$ III, the distributions of SBs and SNRs in the Galaxy are determined by using Monte Carlo simulation, and we study their observational characteristics based on the distributions. Summary of the results is given in $\S \mathrm{IV}$.

$$
\begin{aligned}
& \text { II. PROPERTIES OF THE GALAXY AND METHODS } \\
& \text { OF CALCULATIONS }
\end{aligned}
$$

\section{a) Models}

Statistical studies give a total supernova frequency of 0.02 $\mathrm{yr}^{-1}$ for our Galaxy (Tammann 1977).

Supernovae (SNs) are usually divided into two types. Type I SNs originate from old stellar populations and occur randomly in space and time. We take a frequency for Type I SNs as $0.01 \mathrm{yr}^{-1}$ in the calculations. The frequency of Type II SNs is also taken to be $r_{\mathrm{II}}=0.01 \mathrm{yr}^{-1}$. They are considered as due to evolved massive stars with masses greater than $8 M_{\odot}$. Most of them are bunched in OB associations which concentrate in spiral arms the same as giant molecular clouds and young massive stars do. The number of Type II SNs in a typical OB association $N_{\mathrm{SN}}$ is about 100 (Bruhweiler et al. 1980; Tomisaka, Habe and Ikeuchi 1981). Such a bunching of Type II supernova explosions gives rise to form a superbubble. Since about $f_{\mathrm{OB}}=70 \%$ of all the early-type stars belong to OB associations (Cowie, Songaila, and York 1979), the formation rate of SBs would be

$$
r_{\mathrm{SB}}=r_{\mathrm{II}} f_{\mathrm{OB}} / N_{\mathrm{SN}}=7 \times 10^{-5} \mathrm{yr}^{-1}
$$

The evolution laws of the SNRs and SBs in the ISM are described in Paper I, and their lifetimes $\tau_{\mathrm{SNR}}$, and $\tau_{\mathrm{SB}}$, are also given as

$$
\tau_{\mathrm{SNR}}=1.09 \times 10^{9} T_{0}^{-0.7} n_{0}^{-0.37} \mathrm{yr}
$$

and

$$
\tau_{\mathrm{SB}}=1.02 \times 10^{7} n_{0}^{-0.20} \mathrm{yr}
$$


where $T_{0}$ and $n_{0}$ are the temperature and density of ambient matter. The energy injection of a supernova is set to be $E_{\mathrm{SN}}=10^{51}$ ergs, and energy injection rate in a superbubble is assumed to be $\dot{E}_{\mathrm{SB}}=E_{\mathrm{SN}} / 10^{5} \mathrm{yr}=3.16 \times 10^{38} \mathrm{ergs} \mathrm{s}^{-1}$. It is estimated from equations (2) and (3) that the average number of SNRs and SBs in a steady state to be about $2 \times 10^{4}$ and 700, respectively, for $T_{0}=10^{4} \mathrm{~K}$ and $n_{0}=1 \mathrm{~cm}^{-3}$.

Other parameters are chosen as same as the local ISM: the average radius of diffuse clouds $a=2 \mathrm{pc}$, and their spatial density $N_{\mathrm{cl}}=1.0 \times 10^{-3} \mathrm{pc}^{-3}$. We adopt a flat Galactic rotation curve with circular velocity $V_{\text {rot }}=220 \mathrm{~km} \mathrm{~s}^{-1}$ and neglect any peculiar motions. The width of spiral arms and the half-thickness of the Galactic disk are taken as $500 \mathrm{pc}$ and $200 \mathrm{pc}$, respectively.

Since observations show that the surface brightness of the Galactic disk is an exponential function of galactocentric radius, we also calculate the situations when the density distribution in the disk takes the form

$$
n(R)=n(0) \exp (-R / 3.5 \mathrm{kpc})
$$

We assume that for gas and Type I SNs the distribution begins from $R=0$, while for SBs the distribution begins from the innermost arm region at $R=4 \mathrm{kpc}$. The central gas density $n(0)$ is normalized by the density of solar neighborhood for the gas component. And we fix the supernova and superbubble rates as described above and let them be exponentially distributed in the Galactic disk according to equation (4). As the ambient density is a function of $R$, the evolutionary laws and lifetimes of SNRs and SBs depend on their positions in the Galaxy, and the simple estimation of average steady numbers mentioned above breaks down in this case. The convenient way is to count the actual numbers of them from the results of calculations. However, we are sure that the SNRs and SBs are crowded in the inner region with smaller sizes and shorter lifetimes and rare in the outer region with larger sizes and longer lifetimes. It should be pointed out that the radial scale length of gaseous component is not necessarily the same as that of stellar component. It is quite possible that the scale length of gas distribution is longer than that of stars. But this does not affect our results significantly.

The spiral structure of the Galaxy is very difficult to determine exactly and is subject to considerable debate because the observers inhabit the Galactic disk. Some people believe there are mainly two arms, while some people believe there are four (see Elmegreen 1985 and references therein). Both cases are studied in this work. Two-arm morphology is adopted from the book by Mihalas and Binney (1981) and four-arm morphology from The Cambridge Atlas of Astronomy edited by Audouze and Israel (1988), both of which claim to reproduce well the observed distribution of Galactic $\mathrm{H}$ iI regions.

Monte Carlo method is used for determining the distributions of SNRs and SBs, as Type I SNRs randomly distributing throughout the Galactic disk and SBs concentrating in the spiral arms. At $t=0$ we start without any SNRs and SBs. For each time step, say $10^{4} \mathrm{yr}$ for SNRs and $10^{6} \mathrm{yr}$ for SBs, 100 SNRs and 70 SBs are born in the Galaxy. We assume SNRs are born at random on the disk plane of radius $15 \mathrm{kpc}$, and SBs are born in random along the spiral arm regions with width $500 \mathrm{pc}$. In the case of exponential disk, we use exponential Monte Carlo simulation code to determine the distribution patterns of SNRs and SBs. They expand during the lifetime according to the expansion laws described in Paper I and die at the end of the lifetime.

\section{b) $\mathrm{H}$ I Shells and X-Ray Emission of the Superbubbles}

The $\mathrm{H}_{\mathrm{I}}$ column density of the surrounding shell of an SB is calculated as follows:

$$
N_{\mathrm{HI}}=n_{a} R_{\mathrm{SB}} / 3,
$$

where $R_{\mathrm{SB}}$ is the radius of an expanding SB. The height of an SB is calculated as (Tomisaka and Ikeuchi 1986)

$$
Z_{\mathrm{SB}}=64.3 n\left(Z_{\mathrm{SB}}\right)^{-0.26} \tau_{6}^{0.43} \mathrm{pc}
$$

where $n\left(Z_{\mathrm{SB}}\right)$ is the gas density at $Z_{\mathrm{SB}}$ and $\tau_{6}$ is the age of the SB in units of $10^{6} \mathrm{yr}$. We suppose that the OB associations are all located at $Z=0$, and all the SBs take the form of cylinders which stand perpendicular to the Galactic disk for the sake of simplicity. The latitude range $b$ of the SB with height $Z_{\mathrm{SB}}$ can be easily obtained by knowing the distance $D$ from the Sun as $b= \pm \tan ^{-1}\left(Z_{\mathrm{SB}} / D\right)$.

We get the $\mathrm{H}$ I morphologies at each velocity range $V_{0} \pm \Delta V$ for different lines of sight as shown in Figure 1. According to the $\mathrm{H}_{\text {I }} 21 \mathrm{~cm}$ observations by Heiles (1984), if in a line-of-sight direction with longitude $l$ we find shells of SBs like A, B, C, D and E, we calculate the line-of-sight components of their LSR velocities plus expanding velocities, and if they lie in the velocity range, $V_{0} \pm \Delta V$, with $\Delta V=$ $1 \mathrm{~km} \mathrm{~s}^{-1}$, these parts of shells are plotted. The corresponding radii $R$ of galactocentric circles in Figure 1 to different central velocities $V_{0}$ are

$$
R=\frac{R_{0}}{V_{0} /\left(V_{\text {rot }} \sin l\right)+1},
$$

with $R_{0}$ being the Sun's distance from the Galactic center.

The beamwidth is taken to be $\Delta l=0^{\circ} .36$ in the calculation. For the diffuse $\mathrm{H}_{\mathrm{I}}$ disk, the line-of-sight component of the LSR velocity is taken into account. Further, we add "spatial filters" by which we erase artificially the contribution of background $\mathrm{H}_{\mathrm{I}}$ due to the diffuse $\mathrm{H}_{\mathrm{I}}$ disk at latitudes $|b|>0.5$ to enhance superbubble structures.

We use the cooling function by Raymond, Cox and Smith (1976) as

$$
\Lambda(T)=2.24 \times 10^{-27} T^{0.5} \text { ergs } \mathrm{cm}^{3} \mathrm{~s}^{-1}, \quad T \geq 10^{7} \mathrm{~K}
$$

to calculate the X-ray emissivity. Taking beamwidth as $\Delta l=$ 1.2 , the surface brightness of $\mathrm{X}$-ray emission in a line-of-sight direction is calculated by

$$
E_{\mathrm{X} \text {-ray }}=\int \Lambda(T) n_{c}^{2} d L
$$

in which $n_{c}$ is the gas density of hot cavity with $T \geq 10^{7} \mathrm{~K}$ of SNRs and SBs. The integration includes the contributions 


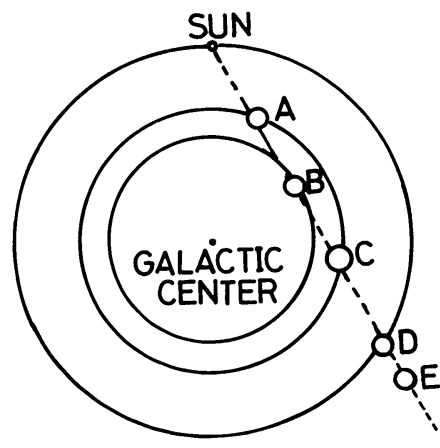

OBSERVED

SPECTRUM

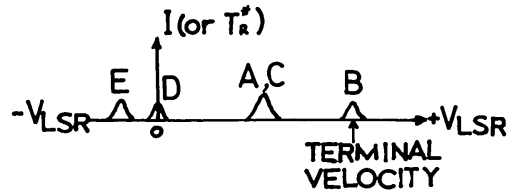

FIG. 1.-A schematic graph which shows the observed region at different radial velocities along a line of sight. The blocks stand for the SBs.

from all SBs and SNRs along the line of sight. For a typical SB with temperature and density to be about $3 \times 10^{7} \mathrm{~K}$ and $10^{-3} \mathrm{~cm}^{-3}$ and radius about $200 \mathrm{pc}$, according to equation (8) the X-ray surface brightness is estimated to be about $1.5 \times 10^{-8} \mathrm{ergs} \mathrm{cm}^{-2} \mathrm{~s}^{-1}$.

III. OBSERVABILITIES OF THE CHIMNEY MODEL

Heiles (1979, 1984) discovered many H I "supershells" with size $0.1-3 \mathrm{kpc}$. Some of them are expanding and some are stationary. In the spatial filtered pictures, they look like worms crawling out of the Galactic disk.

Observations by EXOSAT (Warwick et al. 1985) and Tenma (Koyama et al. 1986) show an X-ray-emitting ridge with $T \geq 10^{7} \mathrm{~K}$ excess above the diffuse X-ray background between at least $l= \pm 40^{\circ}$ toward the galactocentric direction. If we assume a uniform X-ray-emitting disk with radius $10 \mathrm{kpc}$, the average flux would be about $3 \times 10^{-8} \mathrm{ergs}^{-2} \mathrm{~s}^{-1}$ which gives a total X-ray luminosity of about $10^{38}$ ergs $\mathrm{s}^{-1}$. Thermal bremsstrahlung together with iron $K$-line emission at about $6.7 \mathrm{keV}$ seems to fit the spectrum satisfactorily. The scale height of the emission ranges from 100 to $300 \mathrm{pc}$. This is in excess of the contributions from the cataloged discrete sources.

Several possible origins of this $\mathrm{X}$-ray ridge have been proposed. It is obvious that a diffuse hot plasma is not responsible because it can not be confined in the disk. Koyama, Ikeuchi, and Tomisaka (1986) examined the contributions of uncataloged SNRs and concluded that in order to give the observed $\mathrm{X}$-ray luminosity the supernova explosion

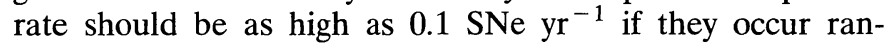
domly. However, if supernova explosions are correlated in space and time, the lifetimes of young X-ray-emitting remnants are prolonged and the necessary rate might be reduced.
We suggest that the above two observed structures are the unified effects of the superbubbles with supershells, surrounding $\mathrm{H}$ i shells of the superbubbles, and an X-ray ridge due to hot X-ray-emitting gas inside the superbubbles. We compare these structures with expected $\mathrm{H}$ i shells and $\mathrm{X}$-ray emission in our chimney model (Norman and Ikeuchi 1989). Several cases for chimney distributions are examined for comparison.

\section{a) Exponential Disk without Spiral Structure}

First, we consider the simple case in which matters are exponentially distributed as in equation (4) but without spiral structure.

A simulated distribution of SNRs and SBs is shown in Figure 2, and the steady numbers of SNRs and SBs are about $10^{4}$ and 500 , respectively.

In Figure 3 (Plates 6-10) some examples of $\mathrm{H}$ i morphologies of different velocity bins are shown. The central velocity $V_{0}$ is taken at interval of $-40 \mathrm{~km} \mathrm{~s}^{-1}$ from $V_{0}=80 \mathrm{~km} \mathrm{~s}^{-1}$ in Figure $3 a$ to $V_{0}=-80 \mathrm{~km} \mathrm{~s}^{-1}$ in Figure $3 e$. The upper panels show the exactly integrated results, and the lower ones are the filtered pictures by which the supershells are seen clearly. Figure 4 (Plate 11) shows the total $\mathrm{H}$ I intensity integrated over all the velocity range with spatial filters. The supershells are quite uniformly distributed in these graphs, and fewer clustering effects are observed. The detailed meanings of these figures and calculated X-ray surface brightness are discussed in $\S$ IIIb.

Next, we investigate the observational characteristics of the SBs with some models of spiral patterns. The real structure of the Galaxy can be understood by comparing these model calculations with observational results.

\section{b) Uniform Disk with Two- and Four-Spiral Arms}

A snapshot of distribution of SNRs and SBs at $t=3 \times 10^{8}$ $\mathrm{yr}$ is shown in Figures $5 a$ and $5 b$ for the two-arm and four-arm situations, respectively, considering the rotation of the Galactic disk. As the lifetime of SBs is much shorter than the rotation time scale, the SBs almost do not leave the arm regions during their lifetimes.

$\mathrm{H}$ I morphologies of five velocity bins the same as in Figure 3 are shown in Figure 6 (Plates 12-16) for the two-arm situation. In Figure 7, we show the galactocentric circles which contribute to each velocity bin $V_{0}$ according to equation (7) for two directions of $l=30^{\circ}$ and $l=330^{\circ}$. From there we can see which part of the disk is seen in each velocity bin.

It is obvious from equation (7) that $R$ is a function of both Galactic longitude and central velocity. When $0<l \leq$ $180^{\circ}$ and $V_{0}>0$, we always see the region with $R<R_{0}$, so the diffuse $\mathrm{H} \mathrm{I}$ is not to be detected between $90^{\circ}<l<180^{\circ}$. When we fix a positive $V_{0}$, as $l$ increases, the detectable $R$ also increases. But there exists a maximum longitude $l_{m}=$ $\sin ^{-1}\left(1-V_{0} / V_{\text {rot }}\right)$ at which the line of sight is the tangent of the galactocentric circle. This corresponds to the edge of the background component in Figure 6. $V_{0}=0$ corresponds to $R_{0}=0$ which is just the solar circle. When $V_{0}<0$, we see the region with $R>R_{0}$. So the diffuse component can be seen at almost all directions. We suppose the $\mathrm{H}$ I disk 


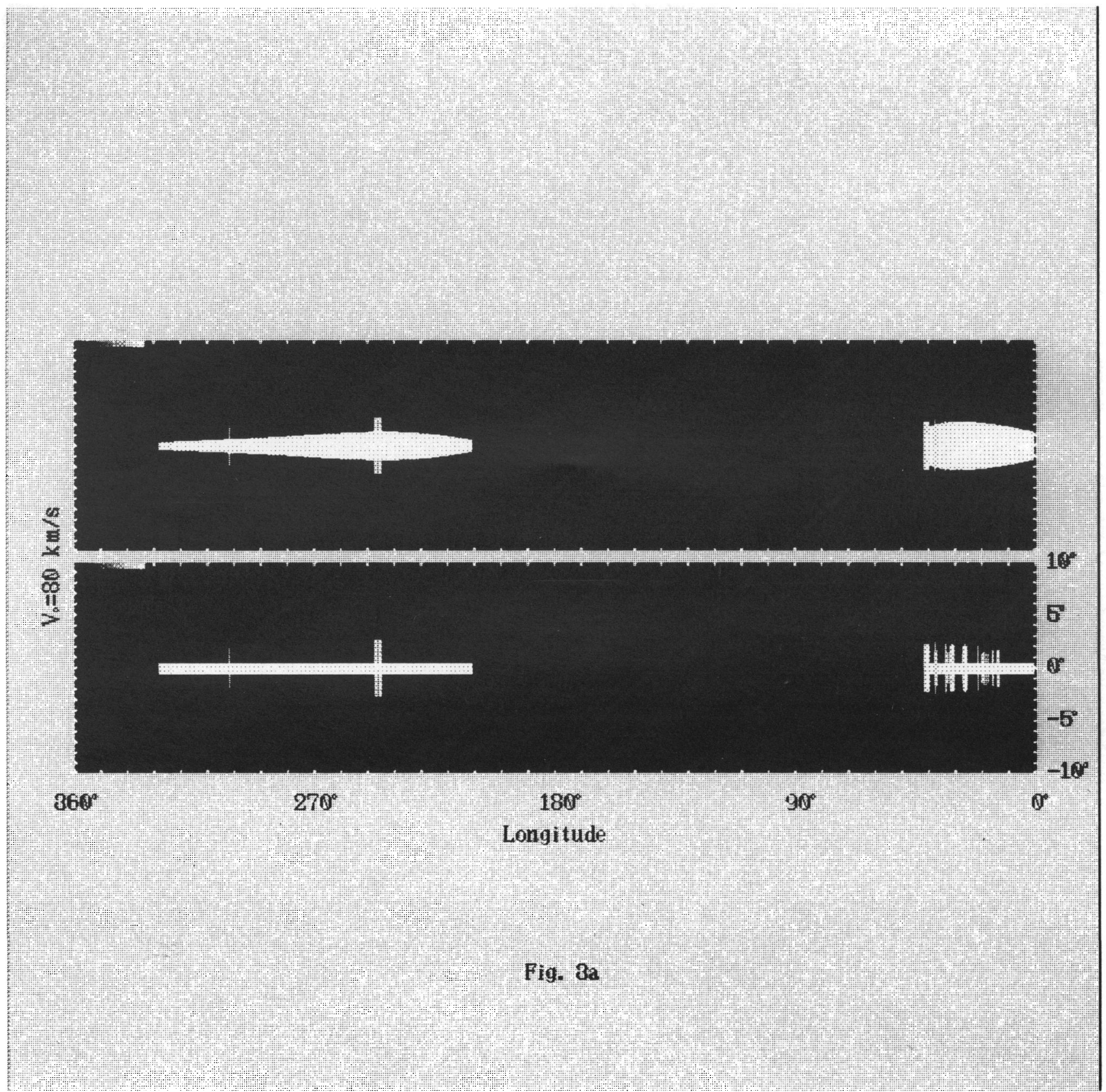

FIG. $3 a$

FIG. 3.-(a) $\mathrm{H}$ I morphology with central velocity $V_{0}=80 \mathrm{~km} \mathrm{~s}^{-1}$ and $2 \mathrm{~km} \mathrm{~s}^{-1}$ wide velocity intervals. The longitude range is from $0^{\circ}$ at the right end of the graph to $360^{\circ}$ at the left end. The latitude range is taken $|b| \leq 10^{\circ}$ from bottom to top, and the beamwidth is $0^{\circ}, 36$. The lower picture is filtered to enhance the supershell structures. (b) $\mathrm{H}_{\text {I }}$ morphology with central velocity $V_{0}=40 \mathrm{~km} \mathrm{~s}^{-1}$. (c) $\mathrm{H}_{\text {I }}$ morphology with central velocity $V^{0}=0$ $\mathrm{km} \mathrm{s}^{-1}$. (d) $\mathrm{H}_{\mathrm{I}}$ morphology with central velocity $V_{0}=-40 \mathrm{~km} \mathrm{~s}^{-1}$. (e) $\mathrm{H}_{\mathrm{I}}$ morphology with central velocity $V_{0}=-80 \mathrm{~km} \mathrm{~s}^{-1}$

Li AND IKEUCHI (see 73, 403) 


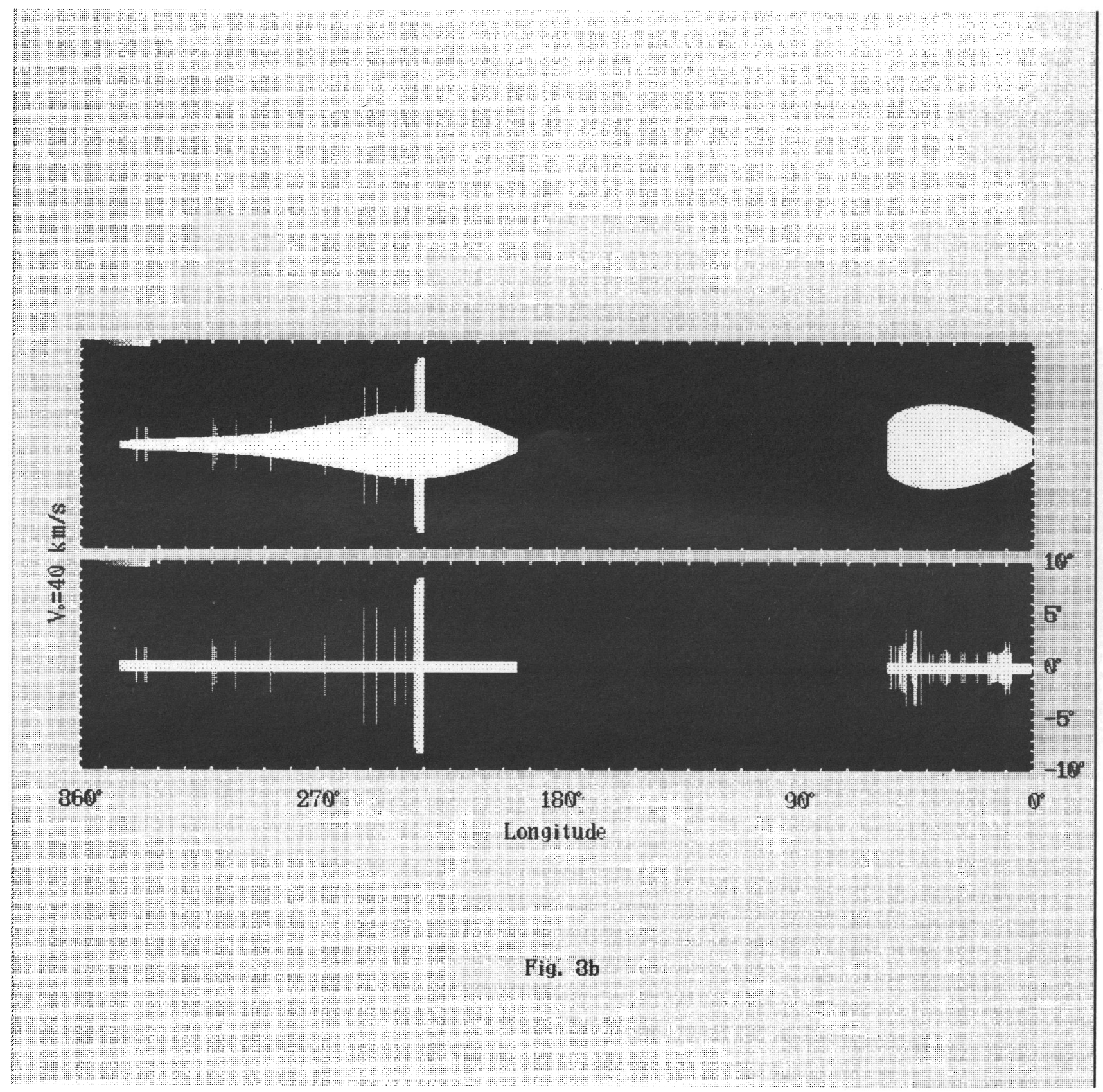

FIG. $3 b$

Li AND IKeUChI (see 73, 403) 


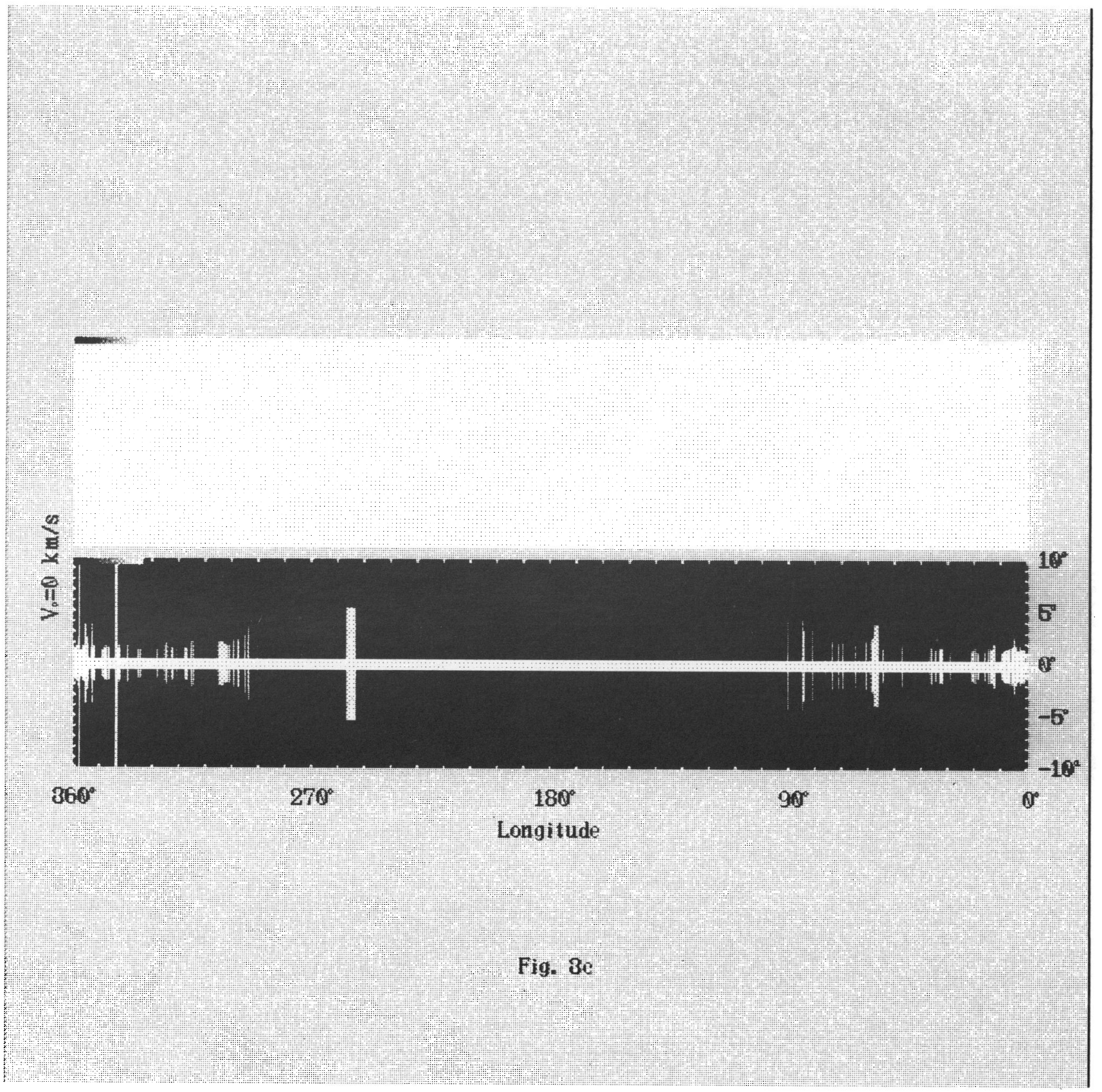

Fig. $3 c$

Li AND IKEUCHI (see 73, 403) 


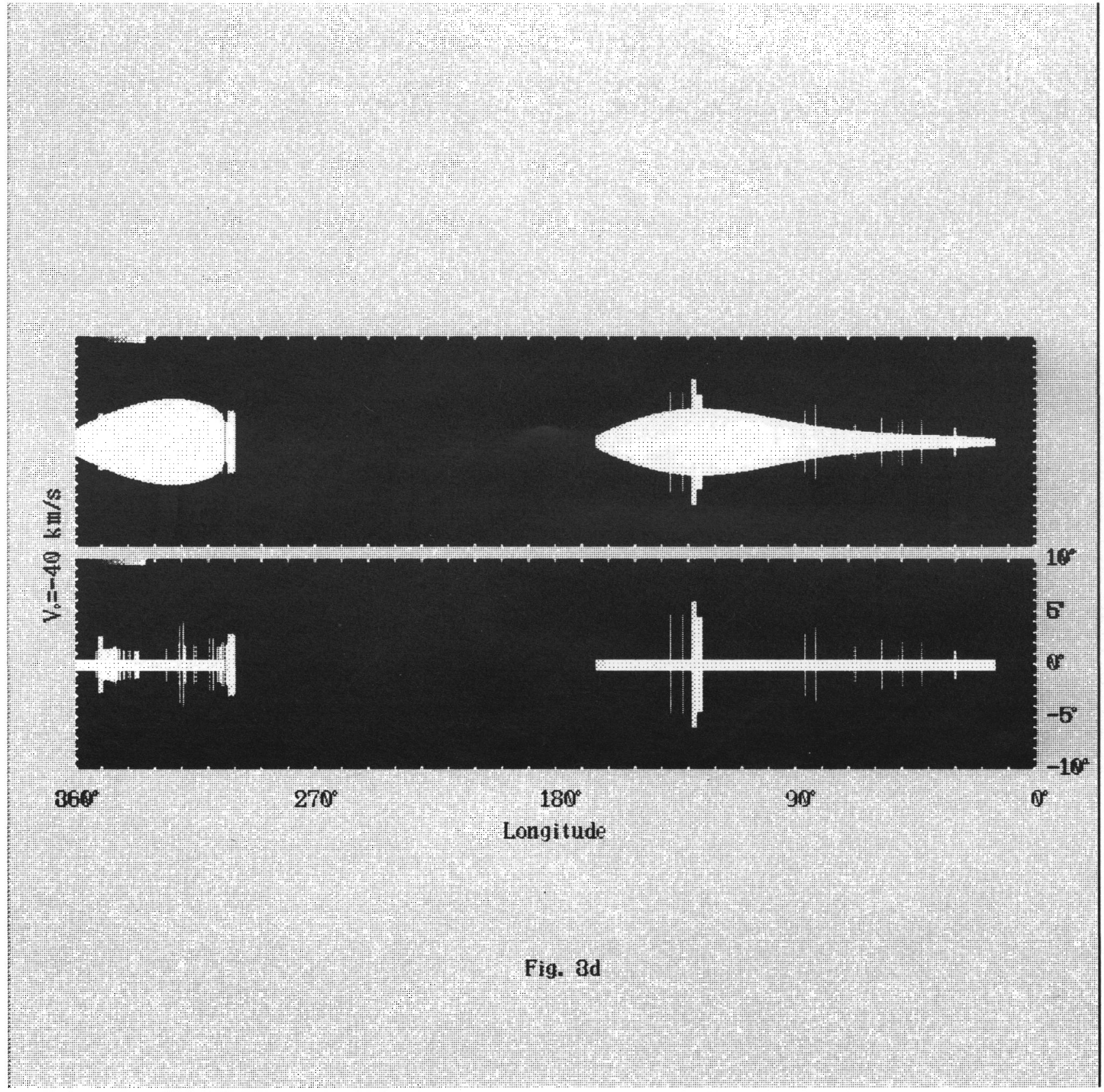

FIG. $3 d$

Li AND IKeUCHI ( 


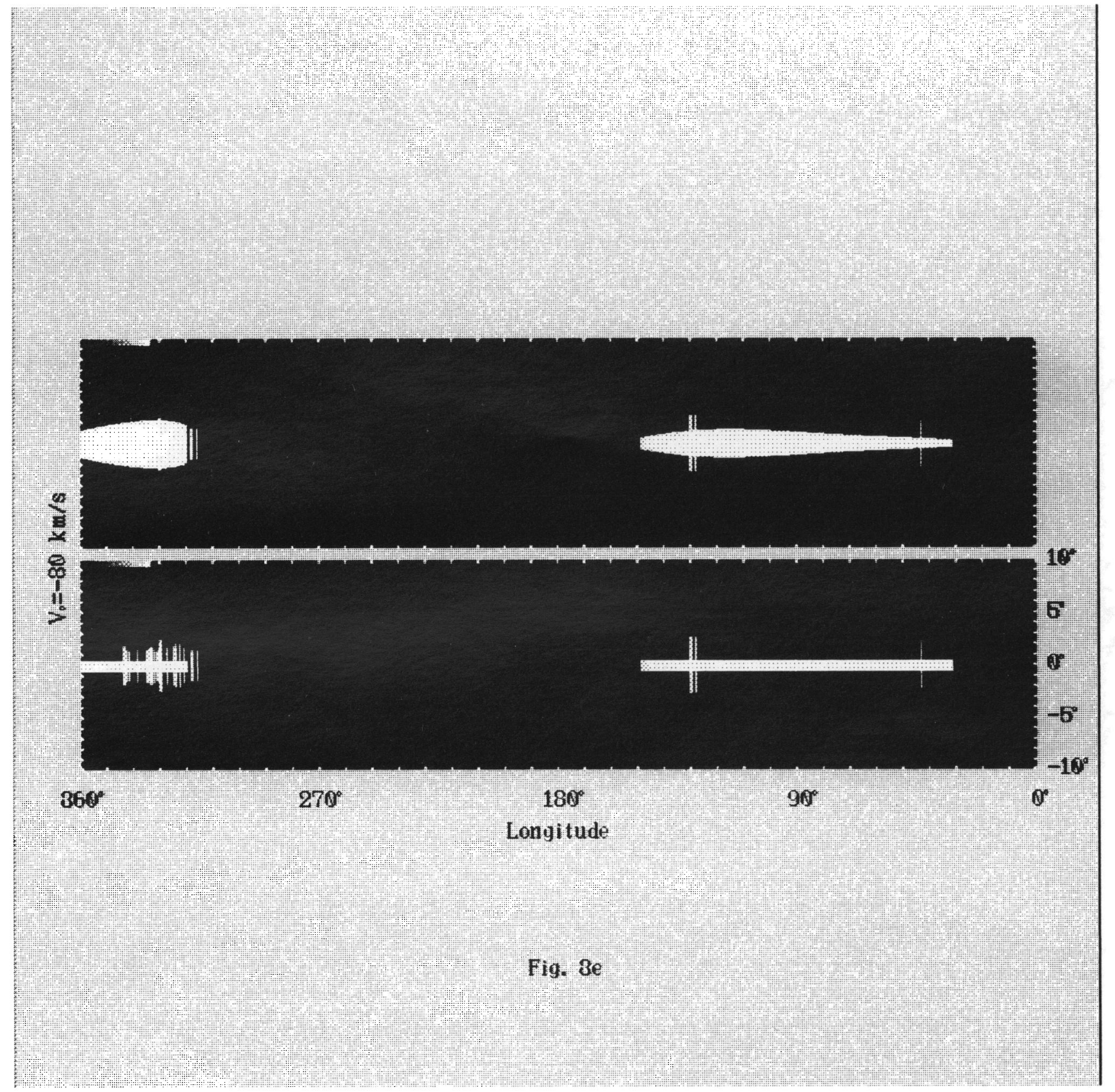

FIG. $3 e$

Li AND IKeUCHI ( 


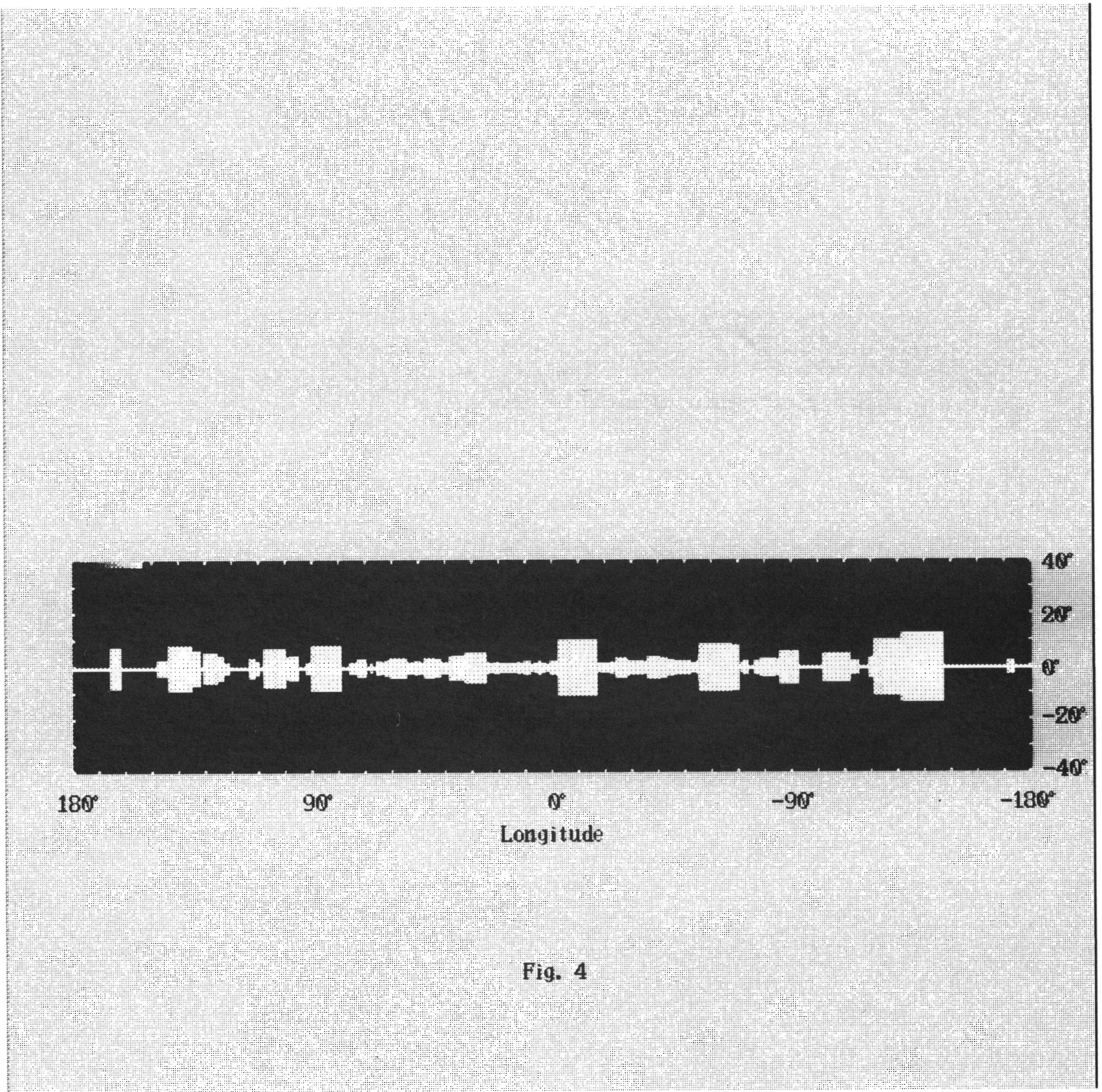

FIG. 4.-Velocity-integrated H I morphology with "spatial filter." The longitude range is from $l=-180^{\circ}$ at the right end to $l=180^{\circ}$ at the left end. The latitude range is $|b| \leq 40^{\circ}$.

Li AND IKEUCHI ( 


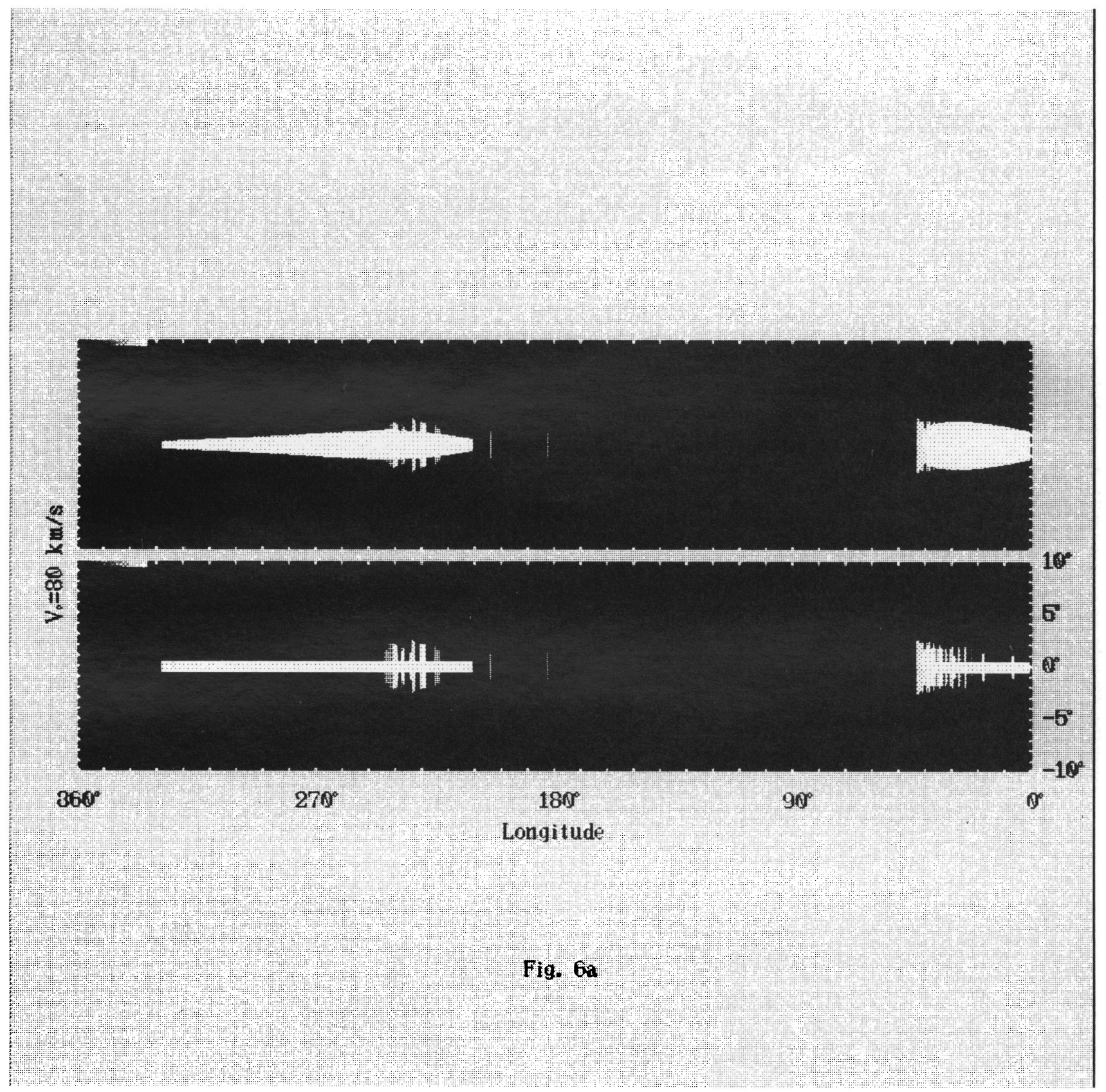

FIG. $6 a$

FIG. 6.-H I morphology with central velocity $V_{0}=80 \mathrm{~km} \mathrm{~s}^{-1}$ and $2 \mathrm{~km} \mathrm{~s}^{-1}$ wide velocity intervals but for uniform disk situation with two-arm spiral pattern. The longitude range is from $0^{\circ}$ at right end of the graph to $360^{\circ}$ at the left end. The latitude range is taken to be $|b| \leq 10^{\circ}$ from bottom to top,

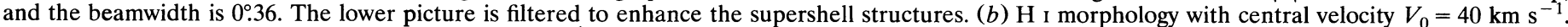
(c) $\mathrm{H}$ I morphology with central velocity $V_{0}=0 \mathrm{~km} \mathrm{~s}^{-1}$. (d) $\mathrm{H}$ i morphology with central velocity $V_{0}=-40 \mathrm{~km} \mathrm{~s}^{-1}$. (e) $\mathrm{H}$ I morphology with central velocity $V_{0}=-80 \mathrm{~km} \mathrm{~s}^{-1}$.

Li AND IKeuchi (see 73, 403) 


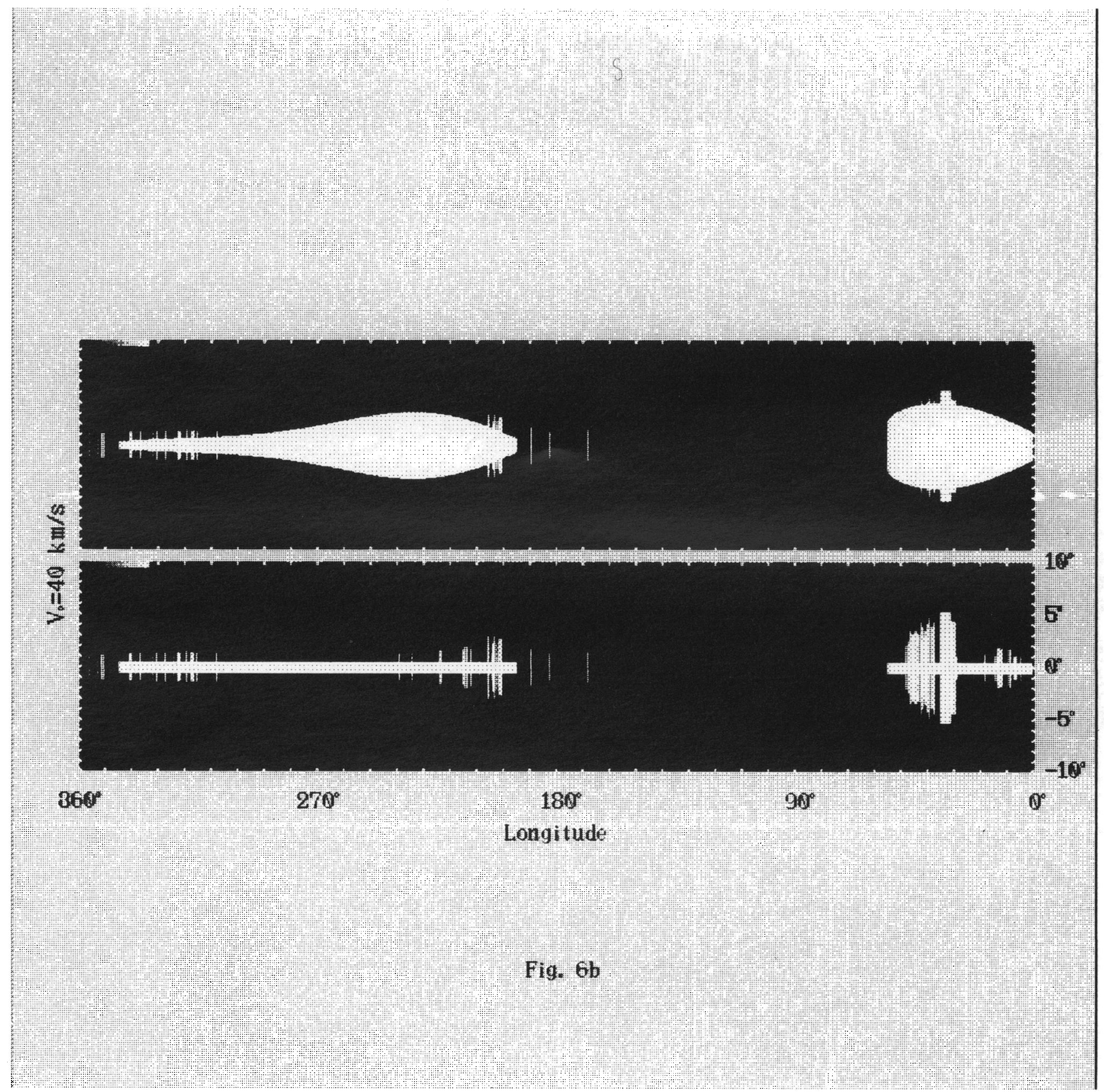

FIG. $6 b$

Li AND IKeuchi (see 73, 403) 


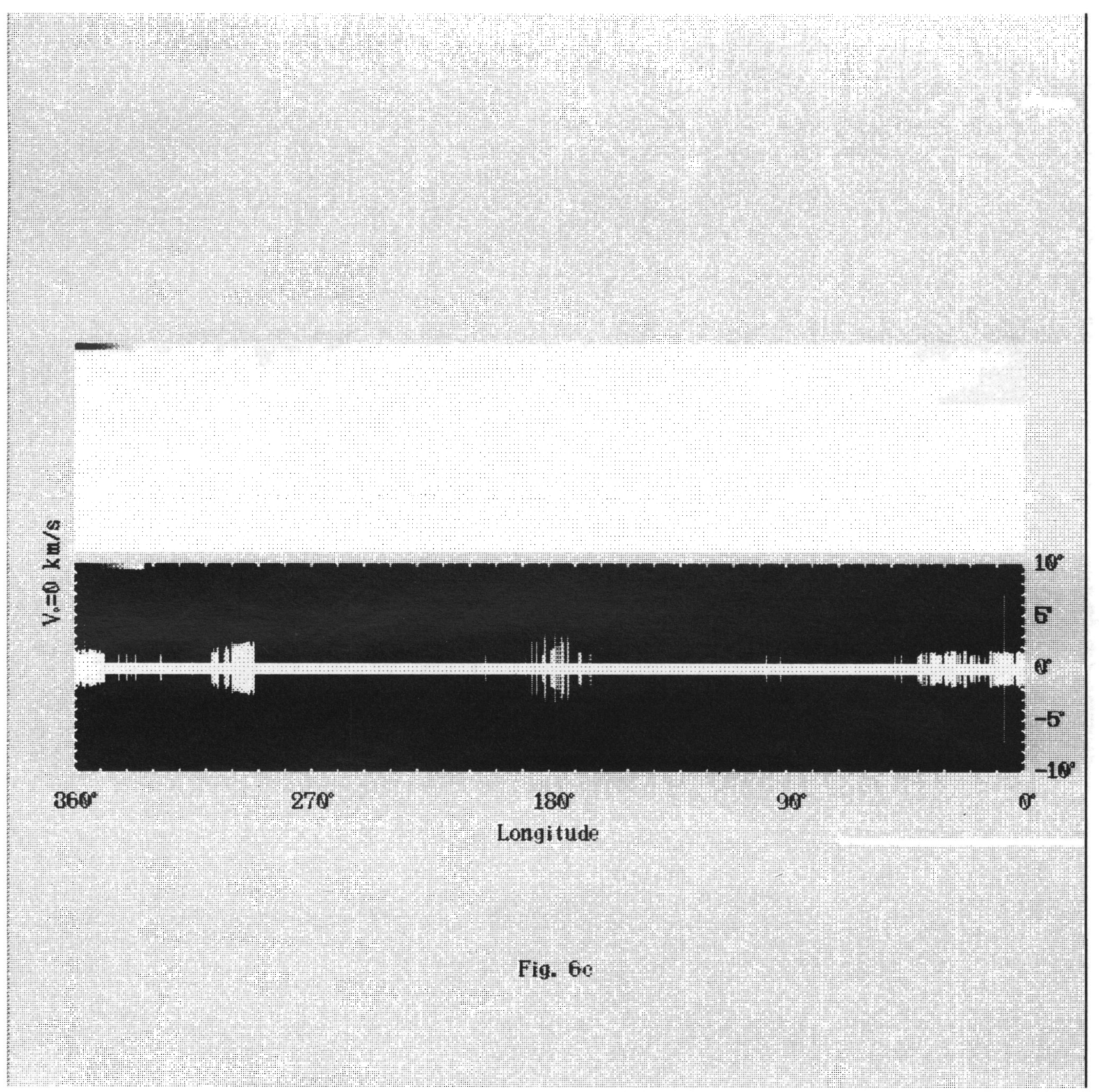

Fig. $6 c$

Li AND IKEUCHI ( see 73, 403) 


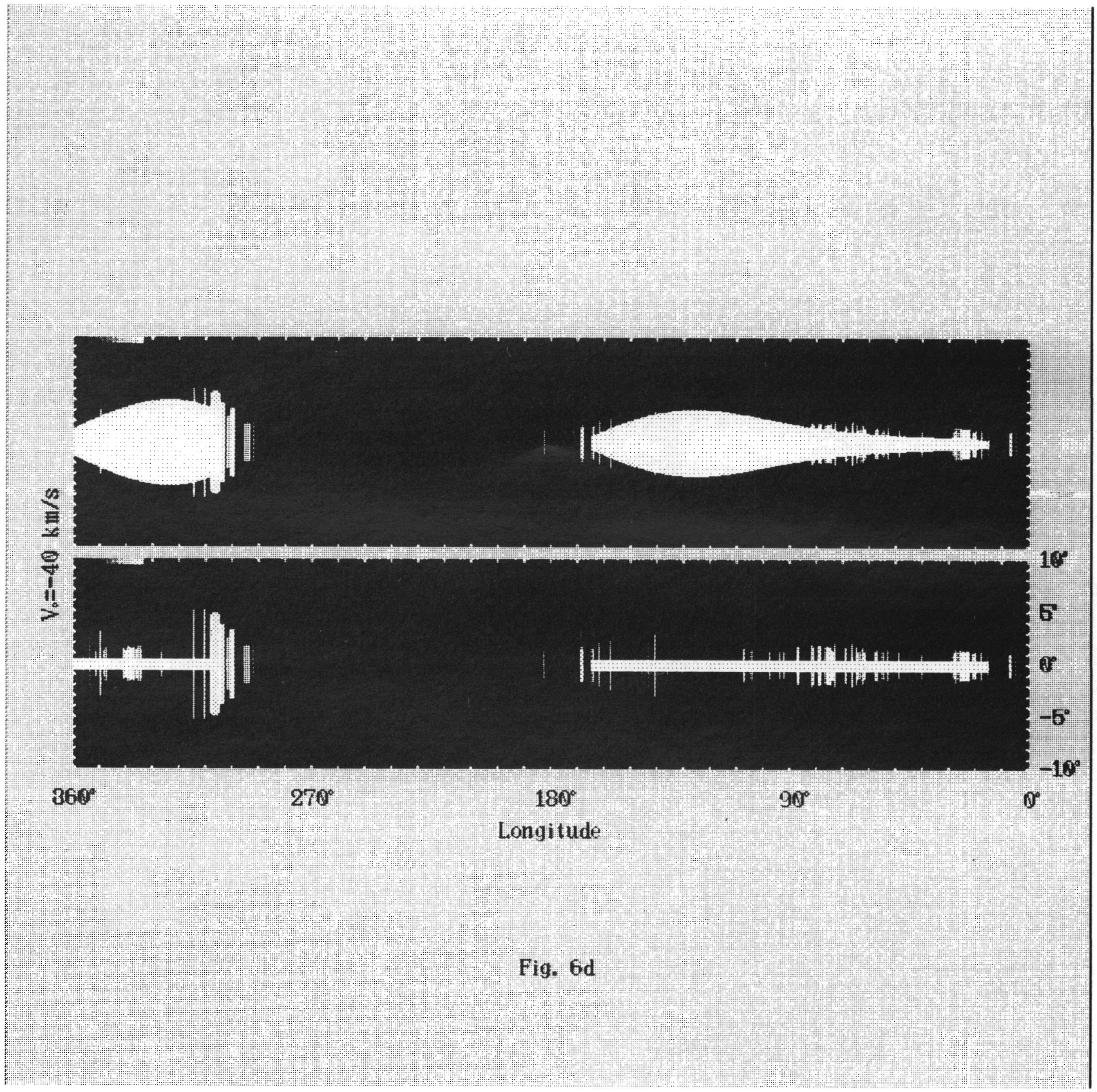

FIG. $6 d$

Li AND IKEUCHI ( 


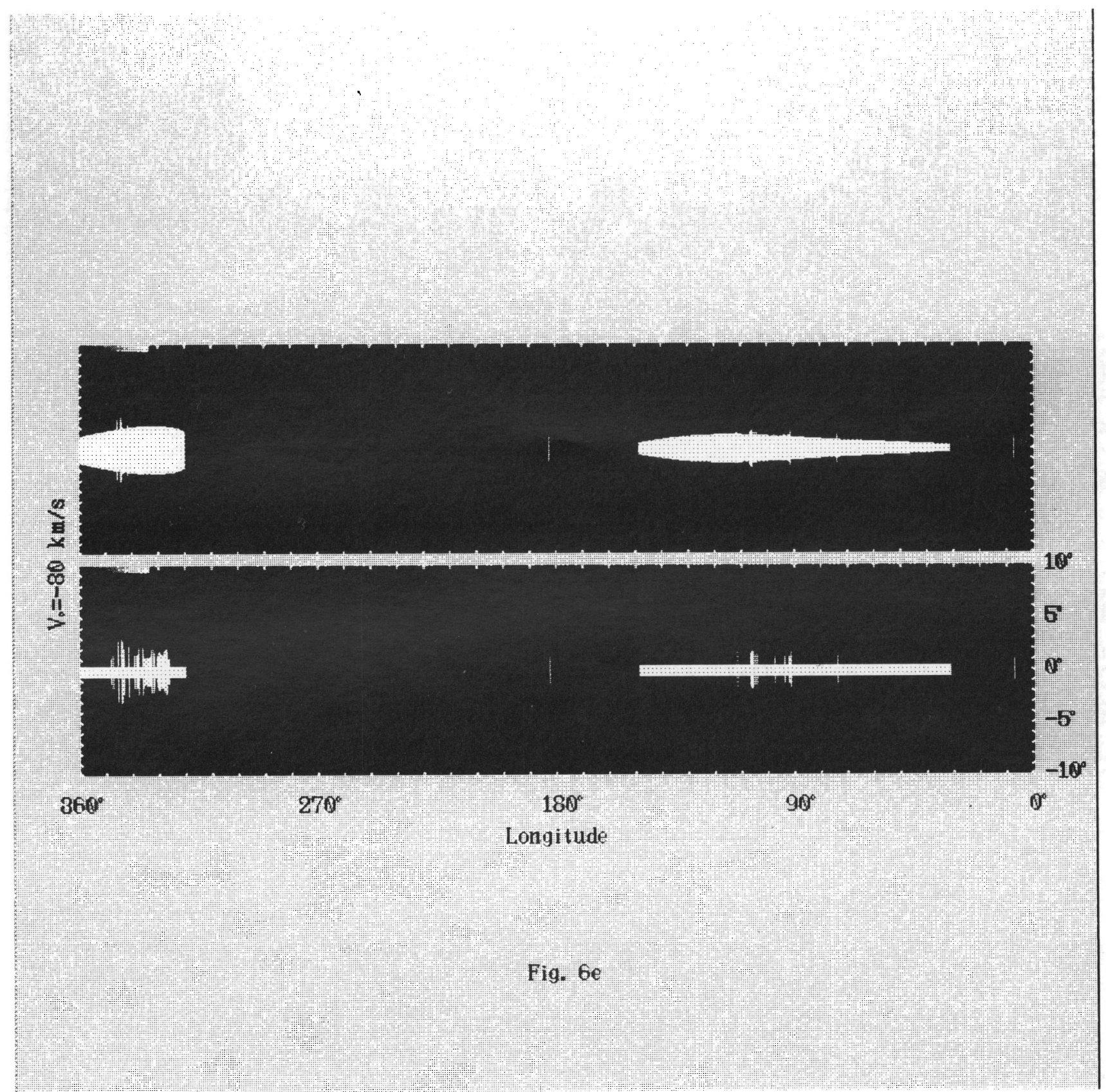

Fig. $6 e$

Li AND IKeuchi ( see 73, 403) 


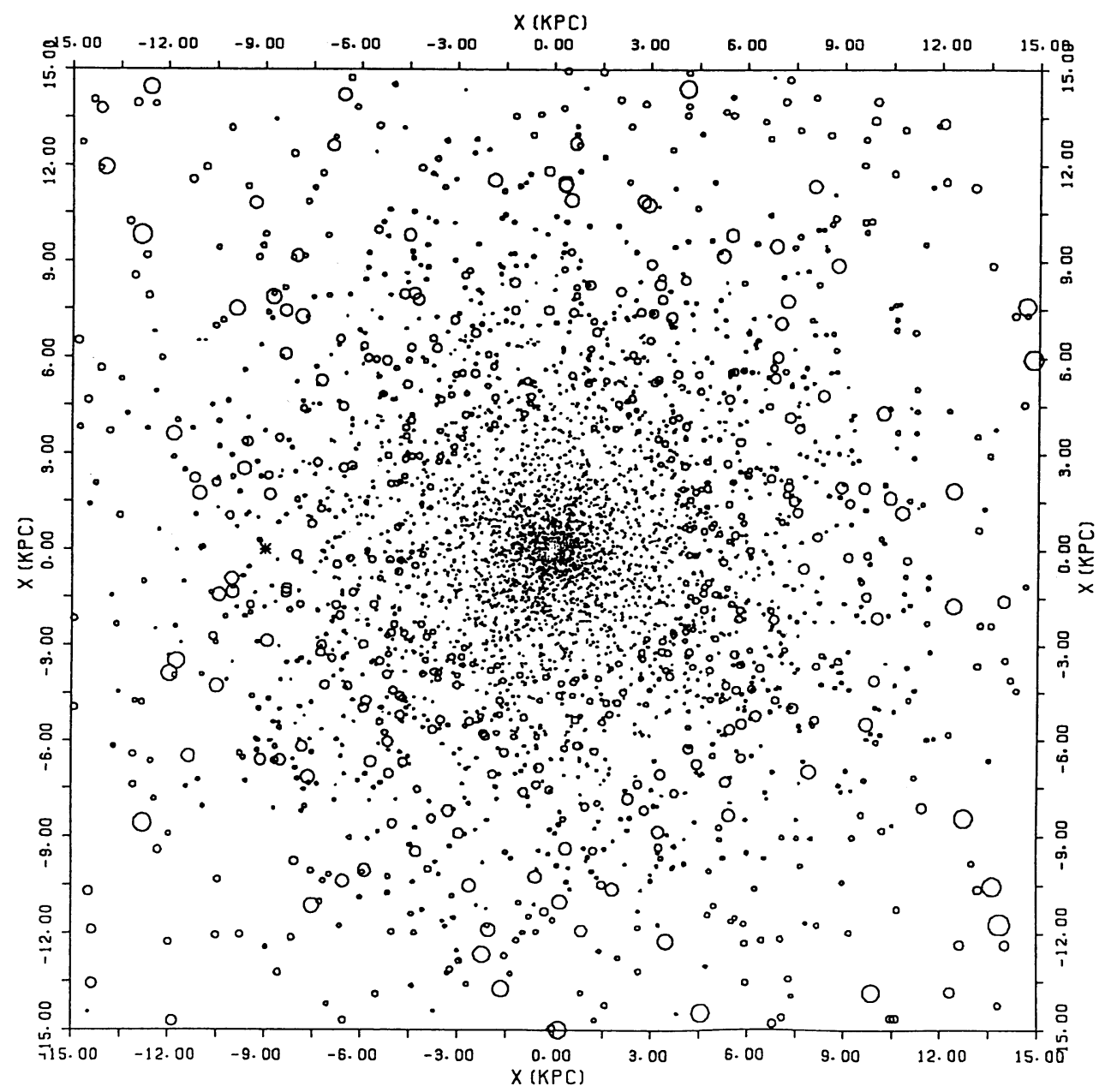

FIG. 2.-A simulated distribution of SNRs and SBs in an exponential disk without any spiral structures by using Monte Carlo method

extends to $R=20 \mathrm{kpc}$ which defines the edge in this situation. When $180^{\circ}<l<360^{\circ}$, the situation is just contrary to the above case as is seen from the figures.

In the upper unfiltered figures, we can see the following characteristics. The $\mathrm{H}$ I emission consists of two components: one is the diffuse component like a broad belt, and the other is the sharp structures standing up from the disk. We call these sharp structures as supershells. The former comes from the diffuse $\mathrm{H}$ I component and the latter from the SBs. The SB contribution shows bunchings at particular longitudes as is clearly seen in filtered figures, because SBs are concentrated in spiral arms. The detectable range in $l$ moves with $V_{0}$ as is seen from the comparison of panels progressing from $(a)$ to $(e)$, since it is limited to $l_{m}=\sin ^{-1}\left(1-V_{0} / V_{\text {rot }}\right)$. Moreover, we can see that the diffuse component becomes wider in latitude with decreasing $\left|V_{0}\right|$, and at $V_{0}=0$ this component comes from all directions. This corresponds to the isotropic background level. Since the contribution at smaller $\left|V_{0}\right|$ arises from the solar neighborhood, the detected angles in latitude become larger. This tendency can be seen also in the heights of supershells. As $\left|V_{0}\right|$ decreases, nearby SBs become detectable and the apparent height $|b|=$ $\tan ^{-1}\left(Z_{\mathrm{SB}} / D\right)$ increases even for the same $Z_{\mathrm{SB}}$. These structures are very similar to the supershells observed by Heiles (1984). The above characteristics of detected $\mathrm{H}_{\mathrm{I}}$ components (limiting $l_{m}$ and height $b$ ) with respect to the observed velocity $V_{0}$ will be confirmed by more detailed observations.

One can notice the differences between the cases with and without spiral structure by comparing Figure 3 with Figure 6 . First, Figure 6 shows preferential longitude regions in each panel to where the $\mathrm{H}$ i shells are prominently concentrated. These correspond to the concentration of SBs in the spiral arms. On the other hand, Figure 3 does not show this kind of preference, and all the directions have equal rights in possessing Hr shells. In other words, the shells are uniformly distributed, and almost no clustering can be observed. Second, in the situations with spiral arms, SBs are restricted in the arm regions and cannot be arbitrarily near the Sun for the spiral patterns adopted as shown in Figure 5. However, in the situation without spiral structure, this restriction does not exist. So the $\mathrm{H}_{\mathrm{I}}$ shells are bigger in Figure 3 than in Figure 6 especially when $\left|V_{0}\right|$ is small.

We perform the same activity for the four-arm situation. From the comparison with Figure 6, we find out the differences between two-arm and four-arm models in the follow- 


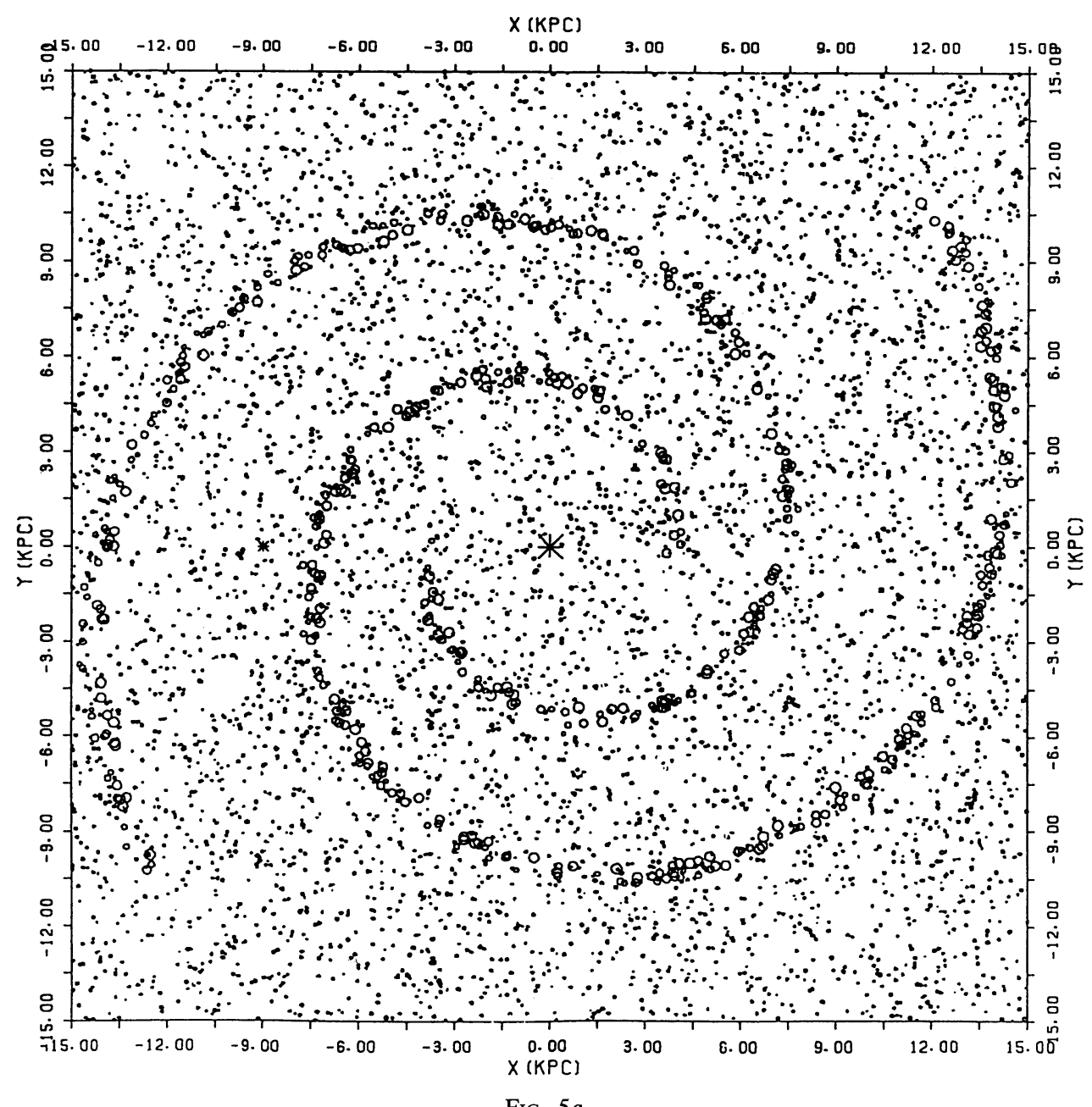

FIG. $5 a$

FIG. 5.-(a) Snapshot of the simulated distribution of SNRs and SBs in uniform disk situation with two-arm spiral pattern. The SBs are concentrated in spiral arm regions. $(b)$ Same as $(a)$ but for the four-arm situation.

ing two points. One is that the number of observed supershells is about 2 times more in the four-arm model than in the two-arm model as is naturally expected. If coarsely averaged, it appears as the number of bunched supershells: one for the two-arm model and two for the four-arm model for a fixed $V_{0}$. The other point is that the height and width of supershells are usually bigger in the four-arm model than those in the two-arm model, because the sites of arms with a given $V_{0}$ are much nearer to the Sun in the former in comparison with the latter. Therefore, we may discriminate two models by comparing the number and structure of supershells in more detailed observations.

These differences are more clearly seen in the velocity integrated $\mathrm{H}_{\mathrm{I}}$ map which includes all the SBs. In Figure 8 (Plate 17), we illustrate it for the two-arm model. The overlapped supershells can be seen around the galactocentric direction because nearby SBs contribute around this direction. In the four-arm model this overlapping is more substantial in the width and height since more arm regions along a line of sight intersect than in the two-arm model.
Comparing with Figure 4, we find out that without spiral structure the density of $\mathrm{H}$ i shells continuously and symmetrically declines on both sides of the Galactic center. However, it is asymmetric on the two sides of the Galactic center in Figure 8 and a gap without $\mathrm{H}$ r shells appears according to the spiral pattern. Furthermore, in Figure 8 the largest shells are concentrated in the Galactic center direction because the nearest arm regions are around this direction. But in Figure 4 the biggest structures come from all directions because the SBs are located at all places, and the same with velocitydivided pictures: the $\mathrm{H}$ I shells are larger in the case without spiral structure than in the case with spiral structure.

The calculated results of X-ray emission of the two-arm situation is shown in Figure 10, with longitude interval $\Delta l=$ 1.2. We can see that the emissivities are typically several times $10^{-8}$ ergs $\mathrm{cm}^{-2} \mathrm{~s}^{-1}$ except for some especially luminous directions of $\sim 10^{-7}$ ergs $\mathrm{cm}^{-2} \mathrm{~s}^{-1}$, where due to the integration effect along the line of sight more than one SB contributes to the X-ray emissivity. There is a very prominent $\mathrm{X}$-ray-emitting ridge compared with no spiral case in 


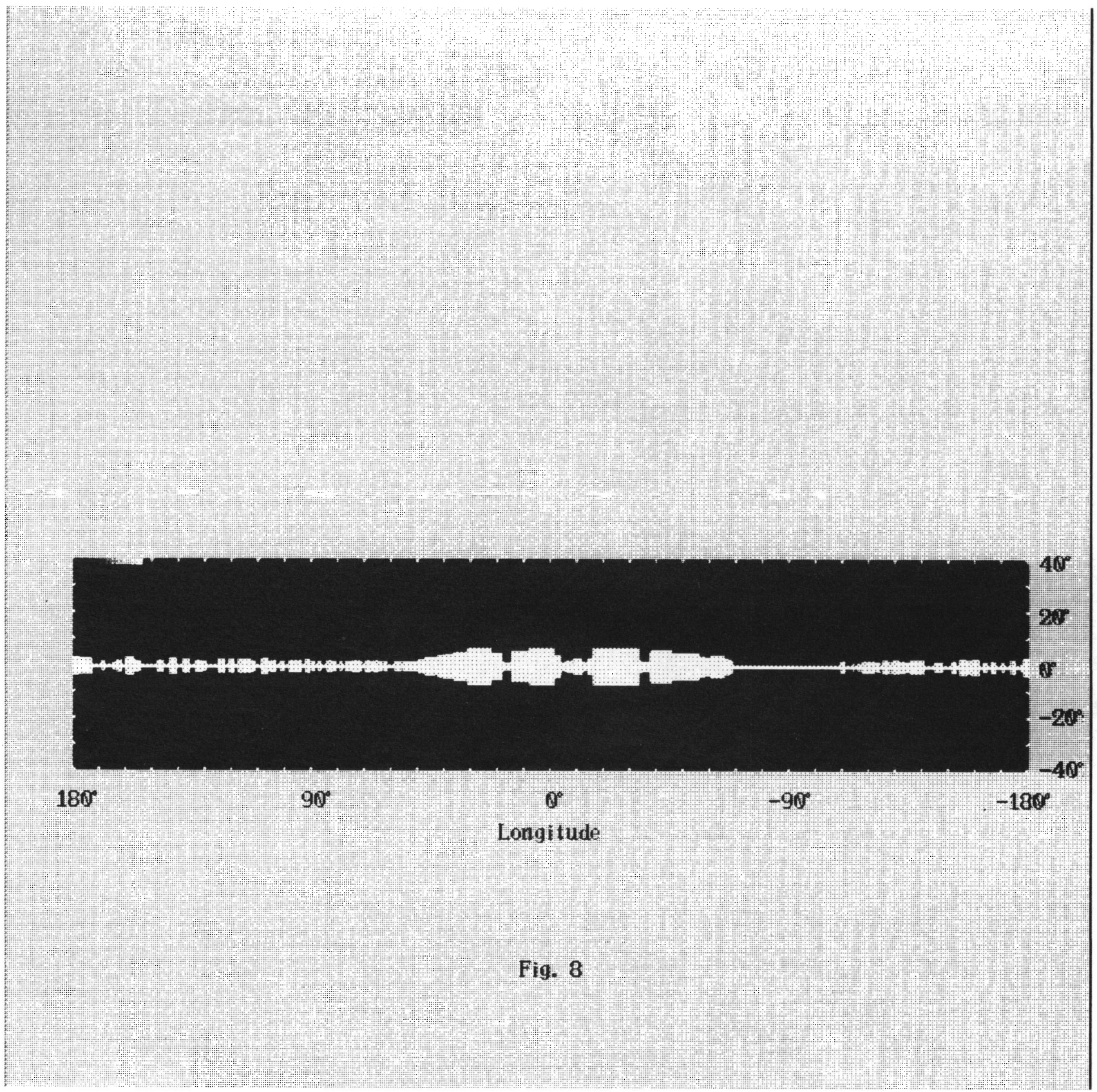

FIG. 8.-Same as Fig. 4 but for uniform disk situation with two-arm spiral pattern

Li AND IKEUCHI (see 73, 405) 


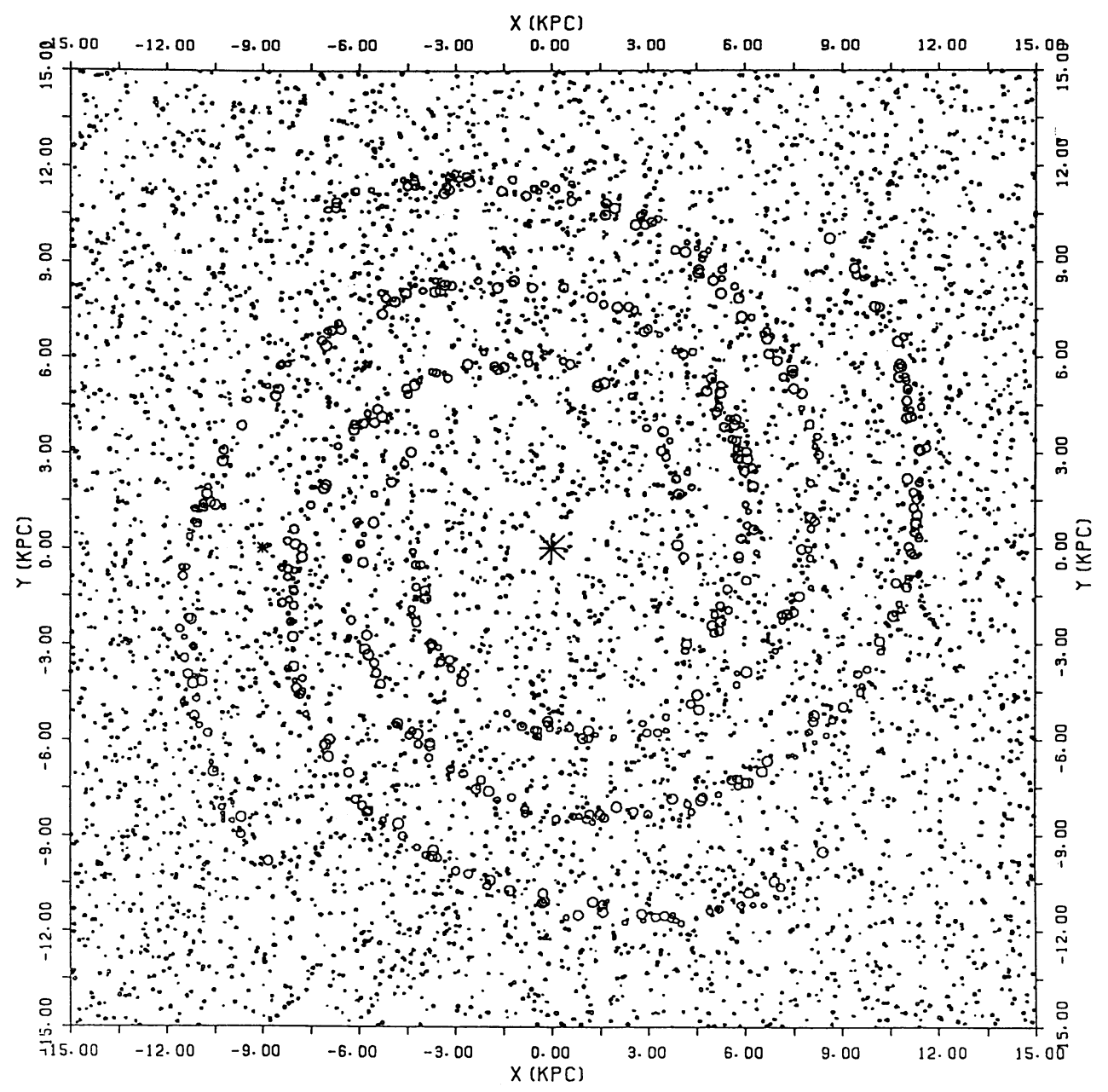

FIG. $5 b$

Figure 9 which is very similar to the exponential disk model profile obtained by Warwick et al. (1985) and which shows a smooth decline of the X-ray intensity on both sides of the Galactic center. The asymmetry with respect to the Galactic center in Figure 9 is the result of random fluctuations of distribution of SBs. Since SBs are much more diffusely distributed in the Galactic disk, Figure 9 does not show a sharp difference of X-ray intensity between on- and off-ridge as in Figure 10. Furthermore, Figure 9 shows fewer fluctuations of the intensity at different longitudes compared with Figure 10 especially on the ridge, which is explained below.

The calculated result shows that in the two-arm situation, the $\mathrm{X}$-ray emission is prominently concentrated in the region $-60^{\circ} \leq l \leq 50^{\circ}$, and at $l \sim-60^{\circ}, l \sim-30^{\circ}$, and $l \sim 40^{\circ}$, further higher peaks of intensity are seen. These correspond to the tangential directions of the arm regions in which the lines of sight intersect with many SBs. Obviously these tangential directions do not exist in no spiral case, so Figure 9 does not show intense fluctuations of X-ray intensity.

The result in the four-arm model is different. In the inner Galactic region, the lines of sight pass through more arm regions, and many more peaks of X-ray emissivity are seen than in the two-arm model. The X-ray-emitting ridge ex- tends to $-80^{\circ} \leq l \leq 60^{\circ}$, which is a little wider than the two-arm model.

In Figure $10 b$, we show the districts where X-ray surface brightness is greater than $10^{-8} \mathrm{ergs} \mathrm{cm}^{-2} \mathrm{~s}^{-1}$ in the Galactic coordinate. It is obvious that these districts are not only prominently concentrated to the inner Galactic longitude region but also confined to the Galactic disk with $|b| \leq 4^{\circ}$. The ridge is generally higher for the four-arm case than for the two-arm case since again the nearby arm regions are closer to the Sun in the four-arm case.

Compared with the results of EXOSAT (Warwick et al. 1985) and Tenma (Koyama et al. 1986) which show that the $\mathrm{X}$-ray ridge extends across $l=-40^{\circ}$ to $40^{\circ}$, our models predict a more extended ridge as $l=-60^{\circ}$ to $50^{\circ}$ in the two-arm model and $l=-80^{\circ}$ to $60^{\circ}$ in the four-arm model. In the situation without spiral structure, the X-ray emission is much more diffusely distributed along the different directions, without sharp difference between the on- and off-ridge. Note that the extent of the ridge in our model is sensitive to the spiral structure. Moreover, the observed intensity distribution is quite smooth, while our results show high fluctuations around the tangential directions of spiral arms. As mentioned in $\S \mathrm{II}$, the spiral structure is not so distinct and 


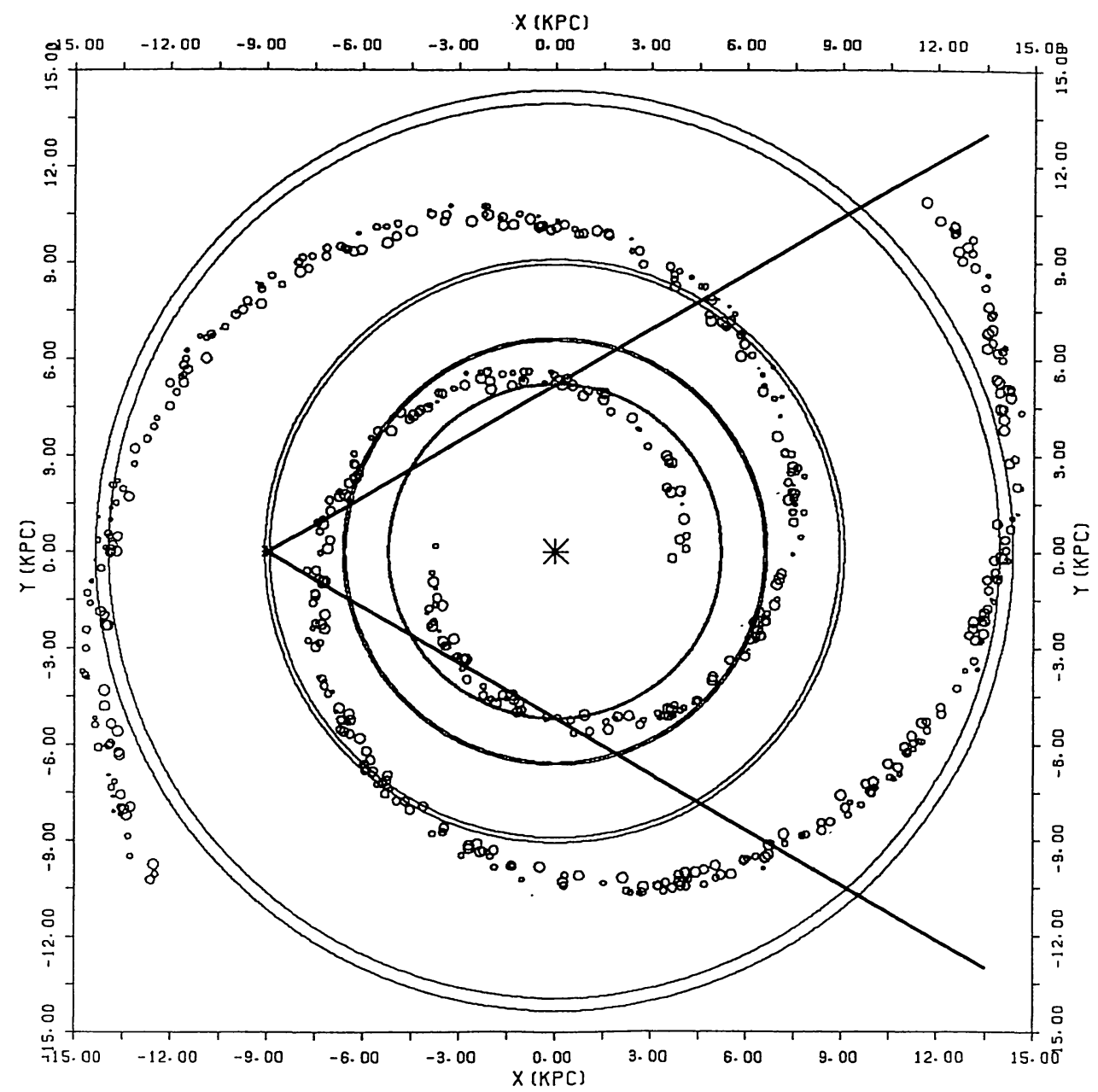

FIG. 7.-Galactocentric circles in Fig. 1 superposed upon Fig. $5 a$ at $l=30^{\circ}$ and $l=330^{\circ}$. Type I SNRs are erased for the sake of clarity. The radii of the circles correspond, outwardly, to $V_{0}=80,40,0$, and $-40 \mathrm{~km} \mathrm{~s}^{-1}$ for $l=30^{\circ}$, and $V_{0}=-80,-40,0$, and $40 \mathrm{~km} \mathrm{~s}^{-1}$ for $l=330^{\circ}$, respectively.

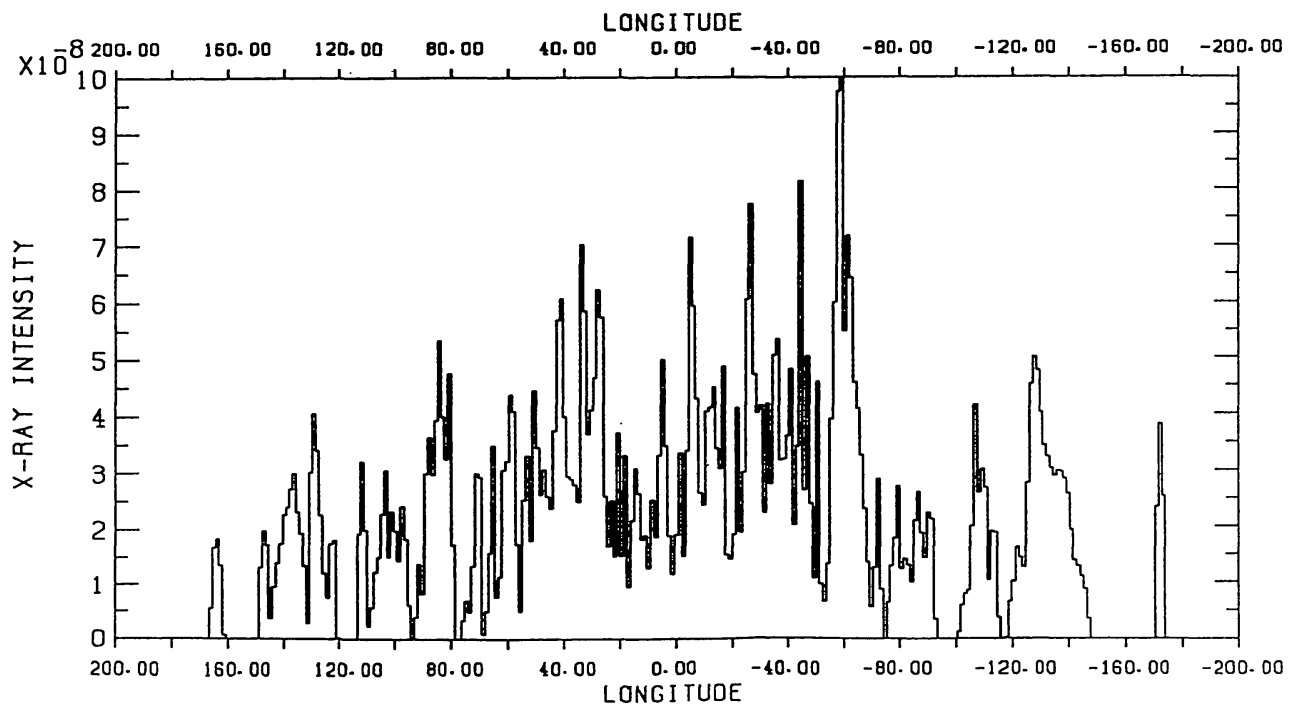

Fig. 9.-Longitude variation of X-ray surface brightness of the SBs along the midplane of the Galactic disk which is exponential but without spiral structure. The beamwidth is taken to be 1.2 . 


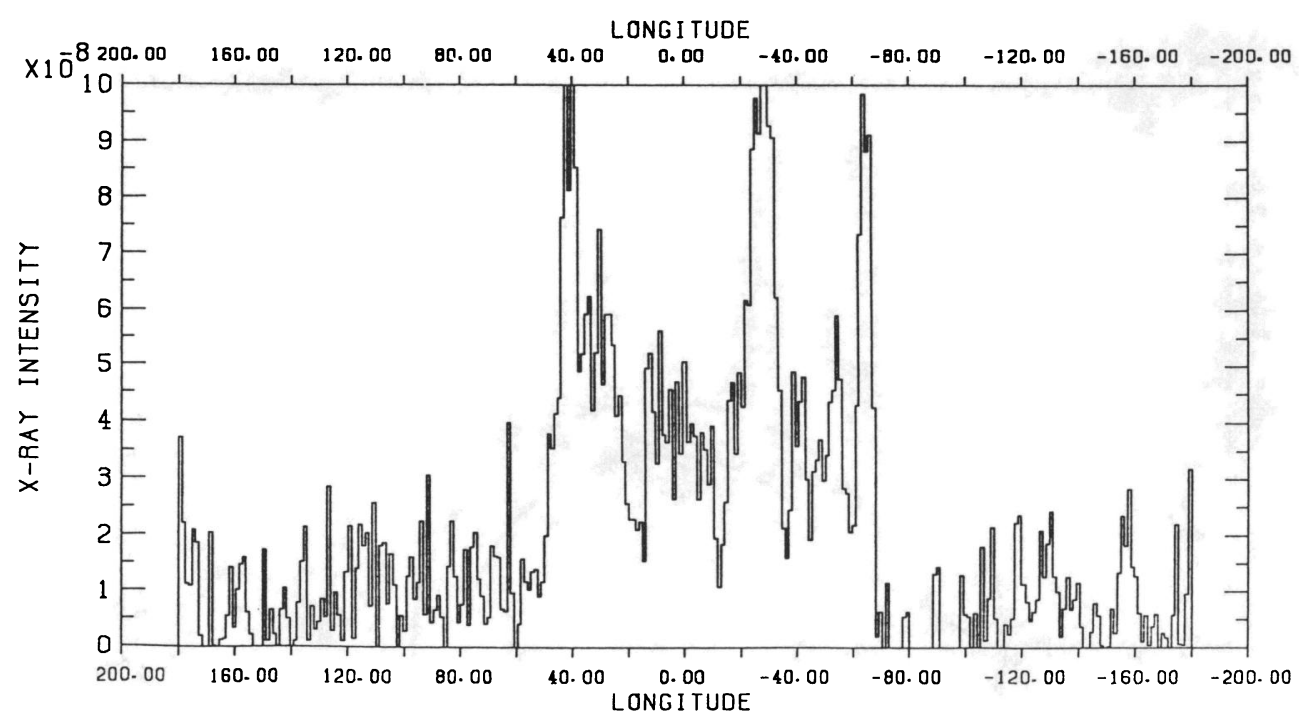

Fig. $10 a$

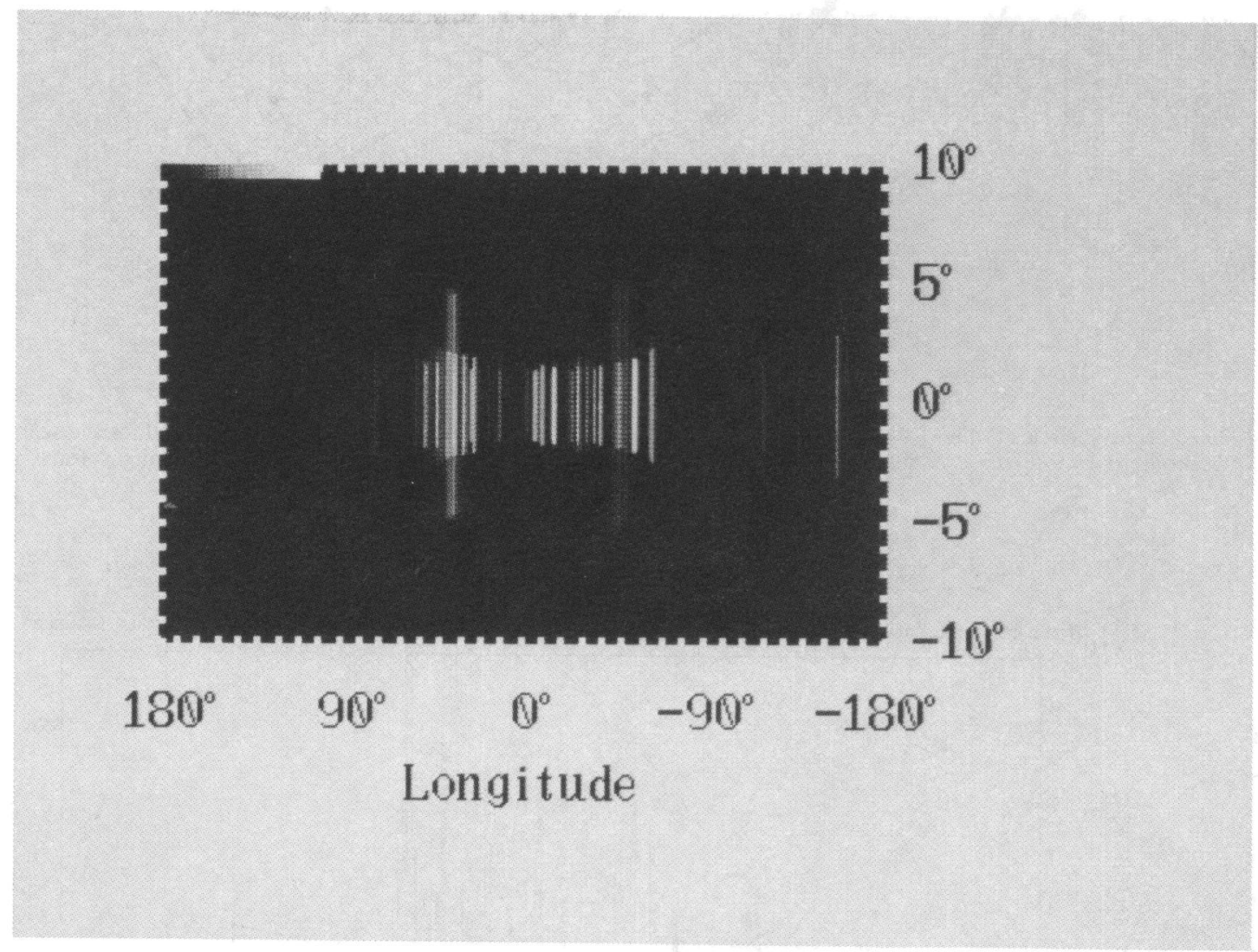

FIG. $10 b$

FIG. 10.- (a) Same as Fig. 9 but for uniform disk situation with two-arm spiral pattern. (b) X-ray surface brightness distribution in the Galactic coordinate. The brightness is proportional to the X-ray intensity.

SBs are probably exponentially distributed in the disk rather than uniformly distributed in the spiral arms. Further, the extra X-ray emission from the Galactic nucleus may arise as indicated by iron $K$-line observations of the X-ray satellite Ginga (Koyama 1989).

\section{c) Exponential Disk with Spiral Structure}

A snapshot of the distribution of SNRs and SBs at $t=3 \times$ $10^{8} \mathrm{yr}$ is shown in Figures $11 a$ and $11 b$ for the two-arm and four-arm situations, respectively. As is expected, SNRs and SBs are distributed exponentially with smaller size, higher 


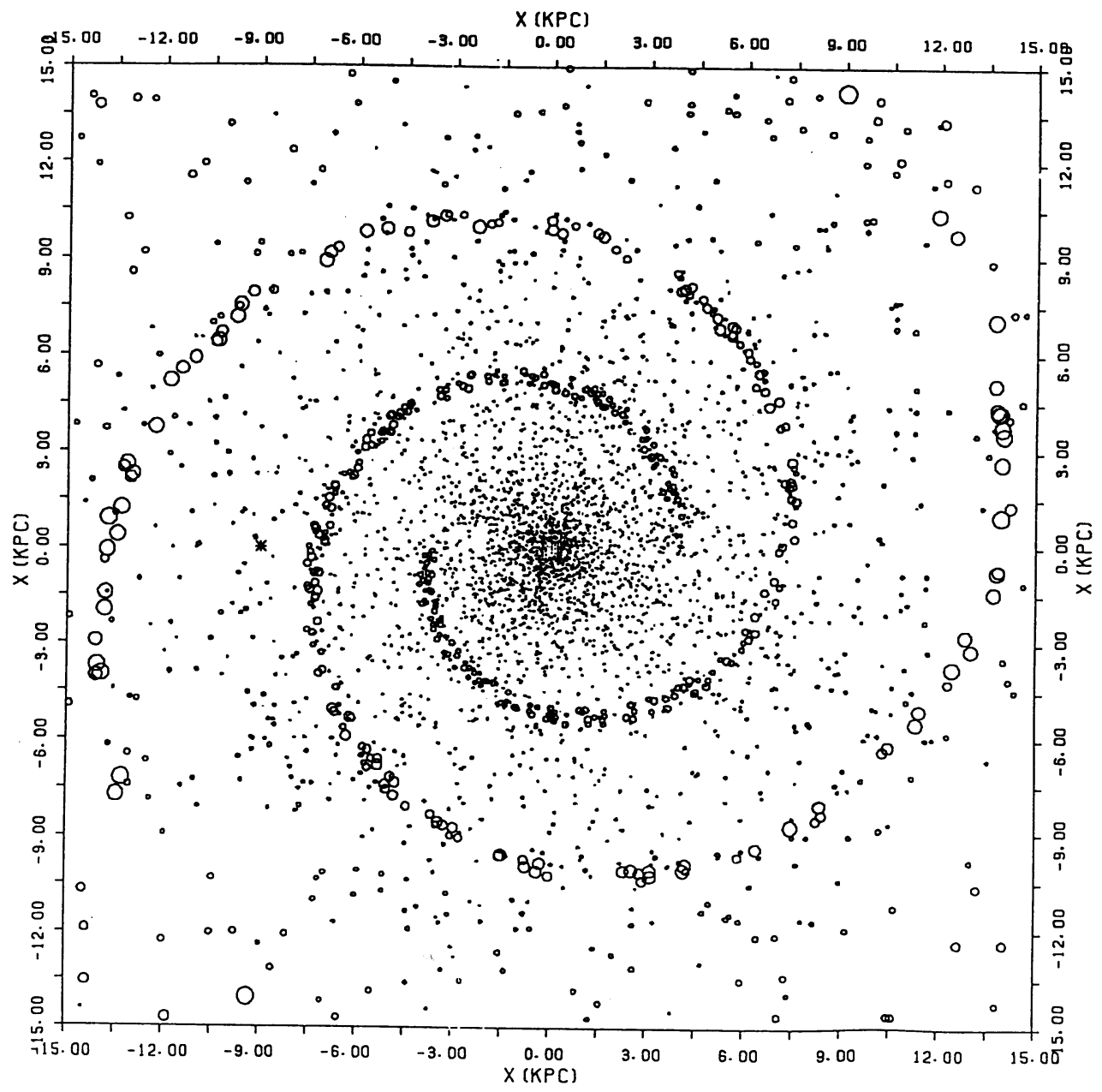

FIG. $11 a$

FIG. 11.- (a) Snapshot of the simulated distribution of SNRs and SBs in exponential disk situation with two-arm spiral pattern. The SNRs and SBs are concentrated in inner Galactic regions. $(b)$ Same as $(a)$ but for the four-arm situation.

concentration in the inner region and larger size, less concentration in the outer region. The steady numbers of SNRs and SBs are about $10^{4}$ and 450 for both the two- and four-arm situations, respectively.

The $\mathrm{H}_{\mathrm{I}}$ maps in five velocity bins are, respectively, shown in Figure 12 (Plates 18-22) for two-arm situation, and the corresponding integrated $\mathrm{H}_{\mathrm{I}}$ maps are shown in Figure 13 (Plate 23).

As is expected, the supershells are further concentrated in the inner Galactic arm regions as is seen in the first Galactic quadrant of Figure $12 a$ and in the fourth quadrant of Figure $12 e$ in addition to the characteristics described in $\S$ III $b$, and the sizes of the shells are smaller in the inner Galactic region and larger in the outer region compared to the uniform disk case. These characteristics are also very clearly seen in the velocity-integrated Figure 13 . We can verify the exponential disk by these features when higher resolution observations are available.

Figure 14 is the result of X-ray emission. This figure shows that the intensities are a little bit lower, but the peak-to-peak fluctuations are smaller compared with uniform disk case.
The reason is that although the number density of SBs is higher around the galactocentric directions, their sizes are very small (about a half time smaller than the uniform disk case). This reduces not only the surface brightness of a single superbubble but also the cross section of detectable SBs along a line of sight. Furthermore, this also reduces fluctuations along different galactocentric directions since the SBs are densely accumulated in the inner regions. However, all these differences are not very prominent. Essential features are similar to the uniform disk case since the spiral structures are same. Since the observed ridge does not show serious fluctuations, the exponential disk seems to conform better to the observations.

\section{d) A Trial Model for the $\mathrm{H}_{\mathrm{I}}$ Holes in M31}

A detailed $\mathrm{H}_{\mathrm{I}}$ survey of M31 shows there are many $\mathrm{H}_{\mathrm{I}}$ holes with sizes from 100 to $1000 \mathrm{pc}$ (Brinks and Bajaja 1986). Similar structures are also observed in M33 (Deul and van der Hulst 1986; Deul and Hartog 1990). We consider these as the top opening of chimneys filled with hot gas when 


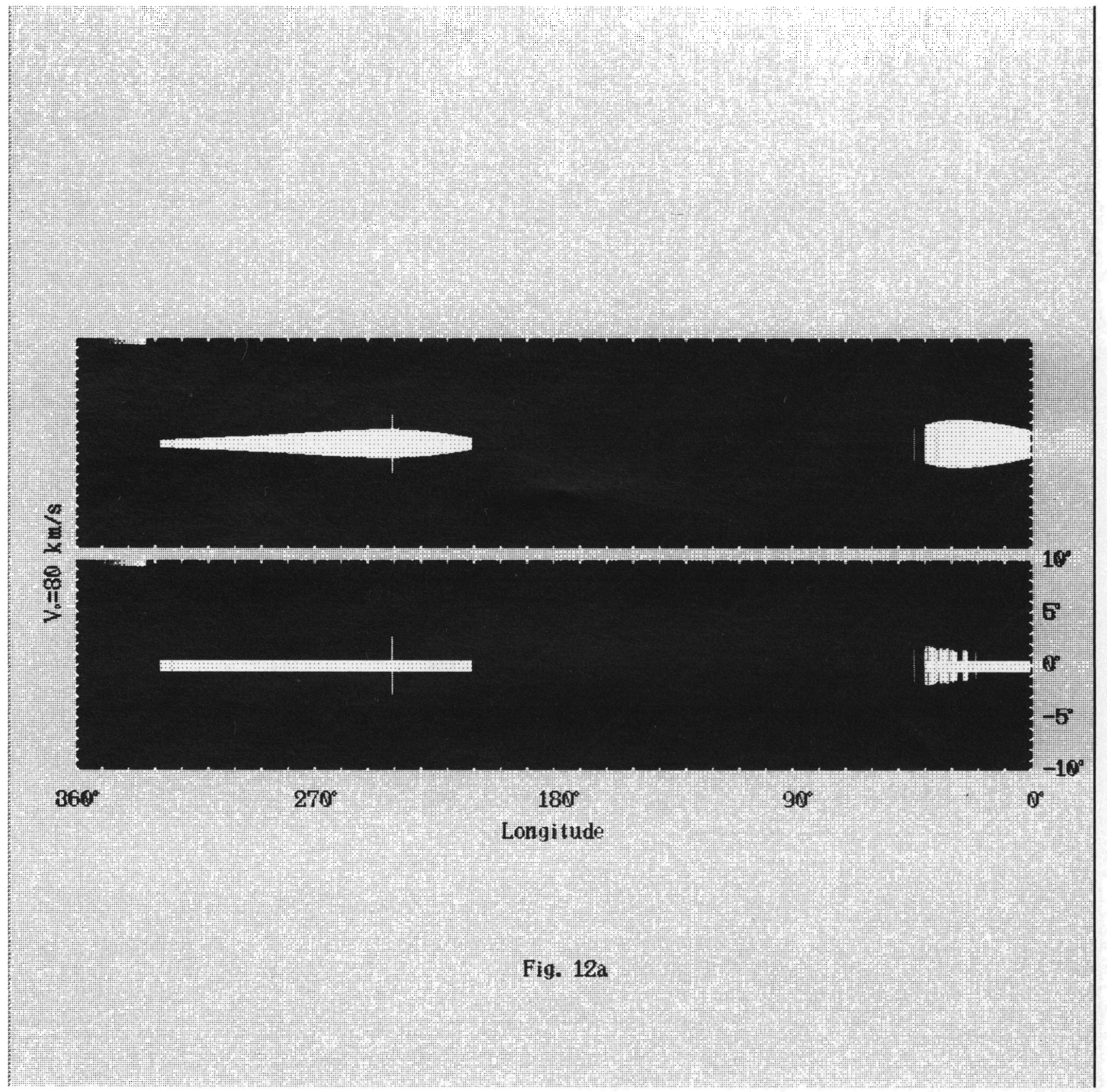

FIG. $12 a$

FIG. 12.-H I morphology with central velocity $V_{0}=80 \mathrm{~km} \mathrm{~s}^{-1}$ and $2 \mathrm{~km} \mathrm{~s}^{-1}$ wide velocity intervals but for exponential disk situation with two-arm spiral pattern. The longitude range is from $0^{\circ}$ at the right end of the graph to $360^{\circ}$ at the left end. The latitude range is taken to be $|b| \leq 10^{\circ}$ from bottom to top, and the beamwidth is $0: 36$. The lower picture is filtered to enhance the supershell structures. $(b) \mathrm{H}$ I morphology with central velocity $V_{0}=40$ $\mathrm{km} \mathrm{s}^{-1}$. (c) $\mathrm{H}$ I morphology with central velocity $V_{0}=0 \mathrm{~km} \mathrm{~s}^{-1}$. (d) $\mathrm{H}$ I morphology with central velocity $V_{0}=-40 \mathrm{~km} \mathrm{~s}^{-1}$. (e) $\mathrm{H}$ I morphology with central velocity $V_{0}=-80 \mathrm{~km} \mathrm{~s}^{-1}$.

Li AND IKEUCHI (see 73, 409) 


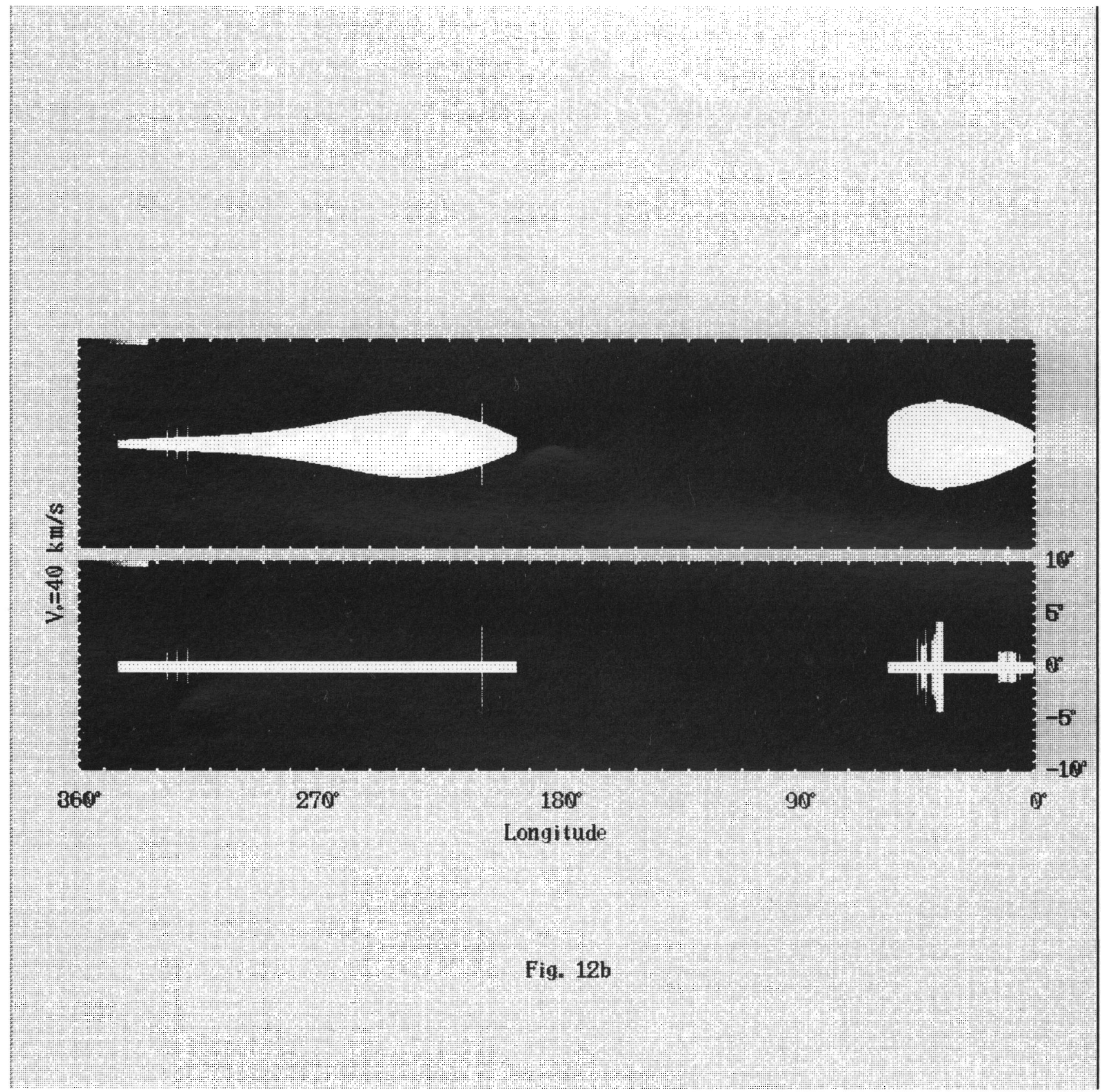

FIG. $12 b$

Li AND IKEUCHI (see 73, 409) 


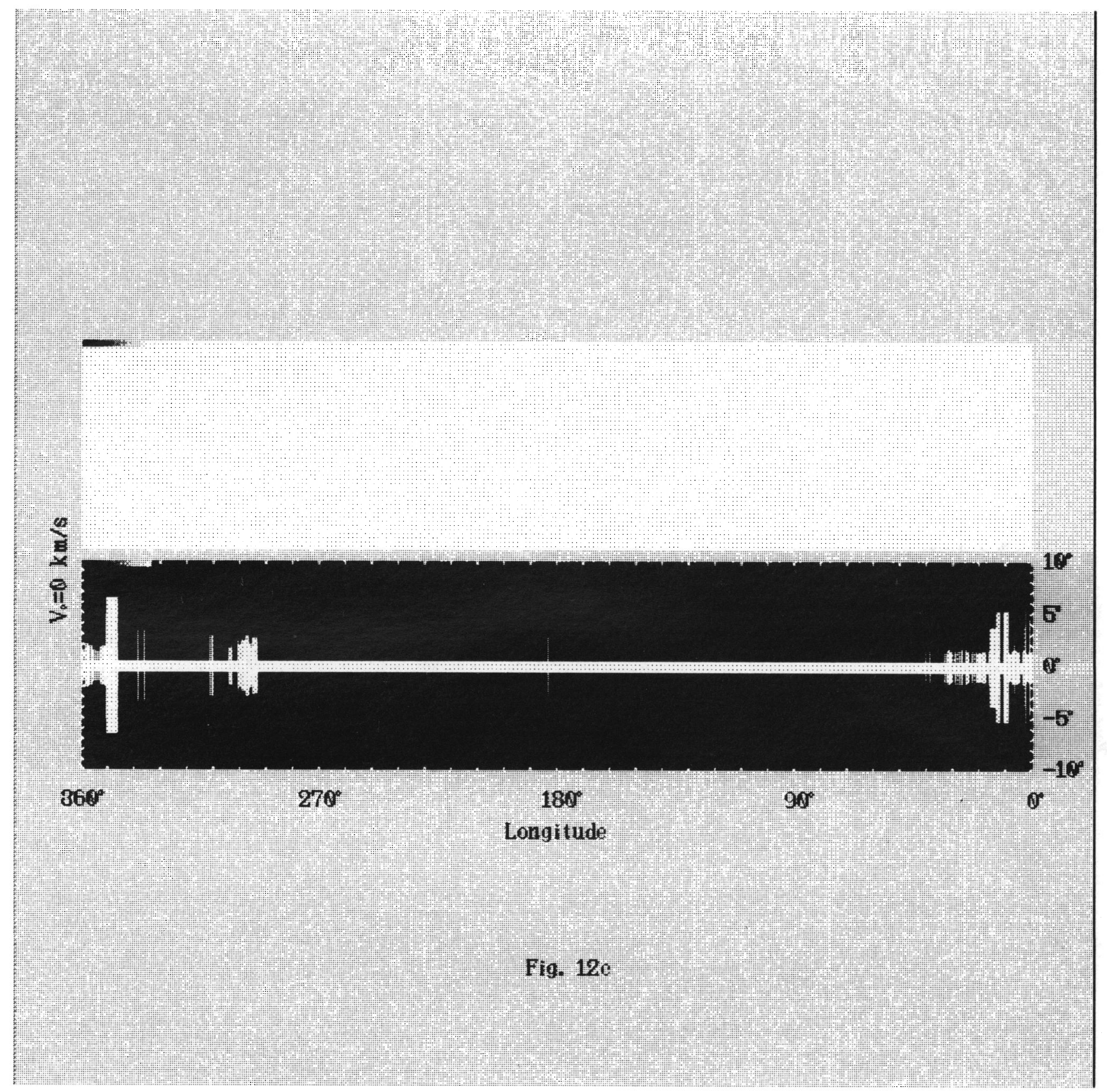

FIG. $12 c$

Li AND IKEUCHI (see 73, 409) 


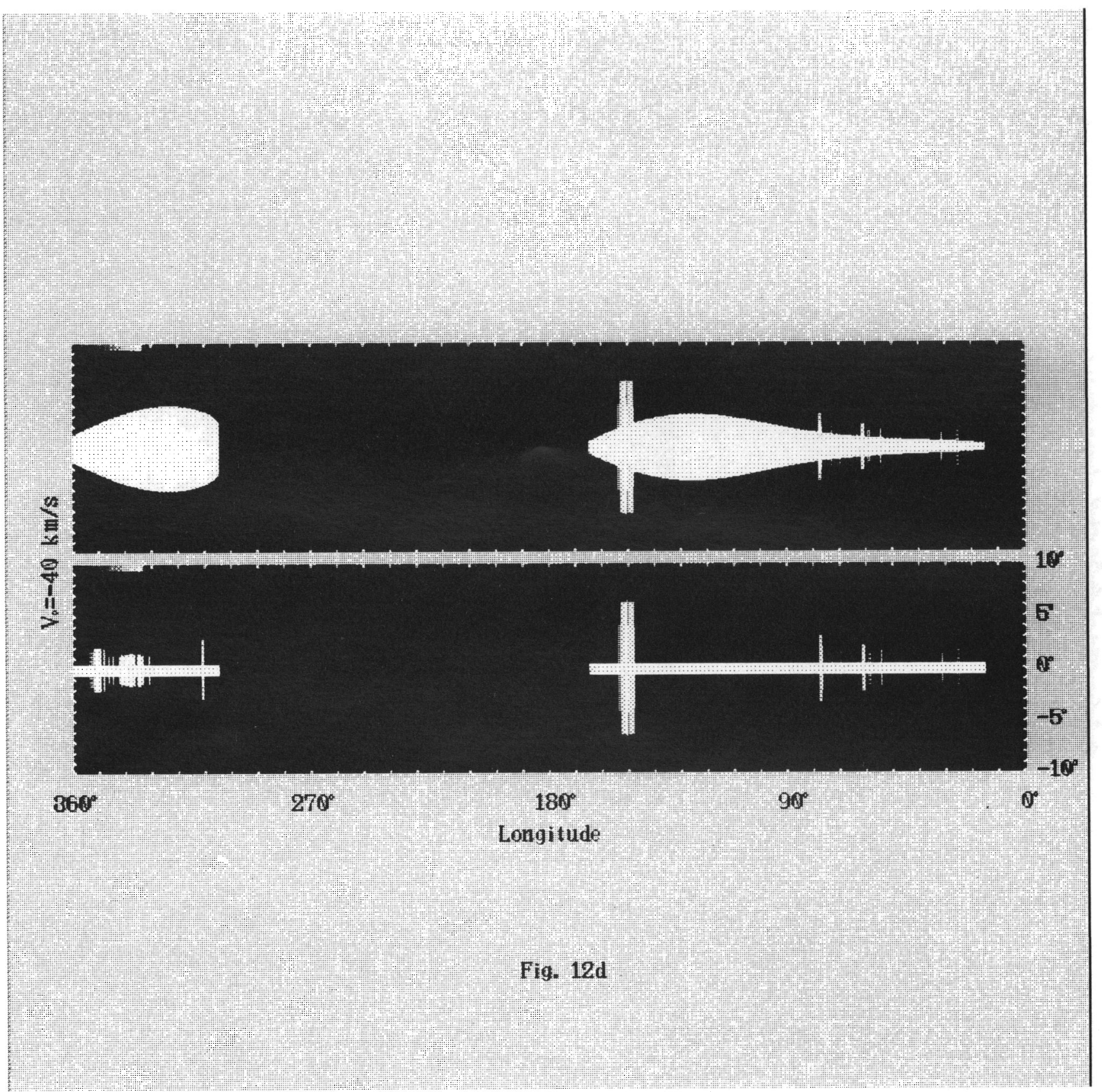

FIG. $12 d$

Li AND IKEUCHI (see 73, 409) 


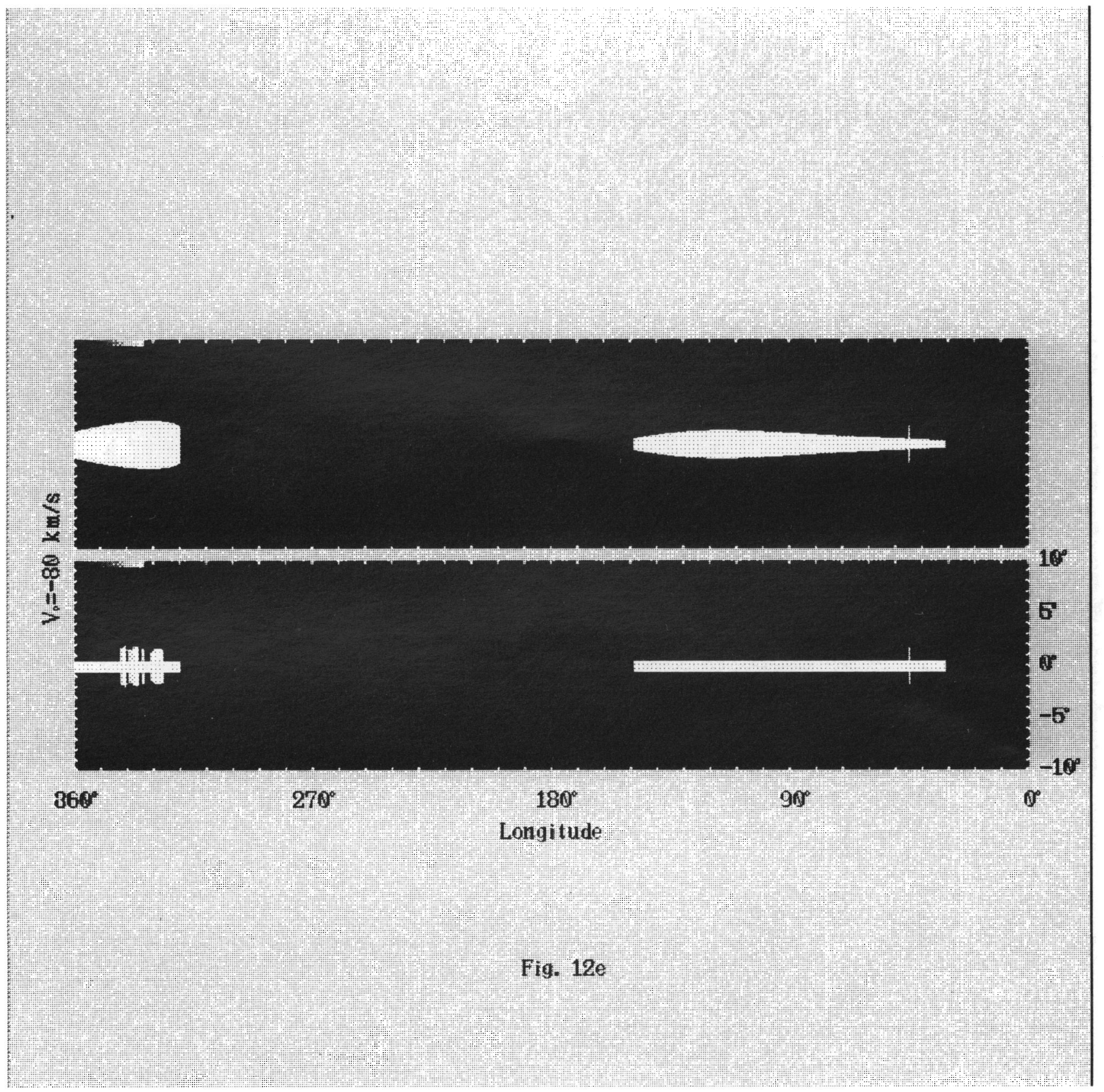

FIG. $12 e$

Li AND IKeUChI (see 73, 409) 


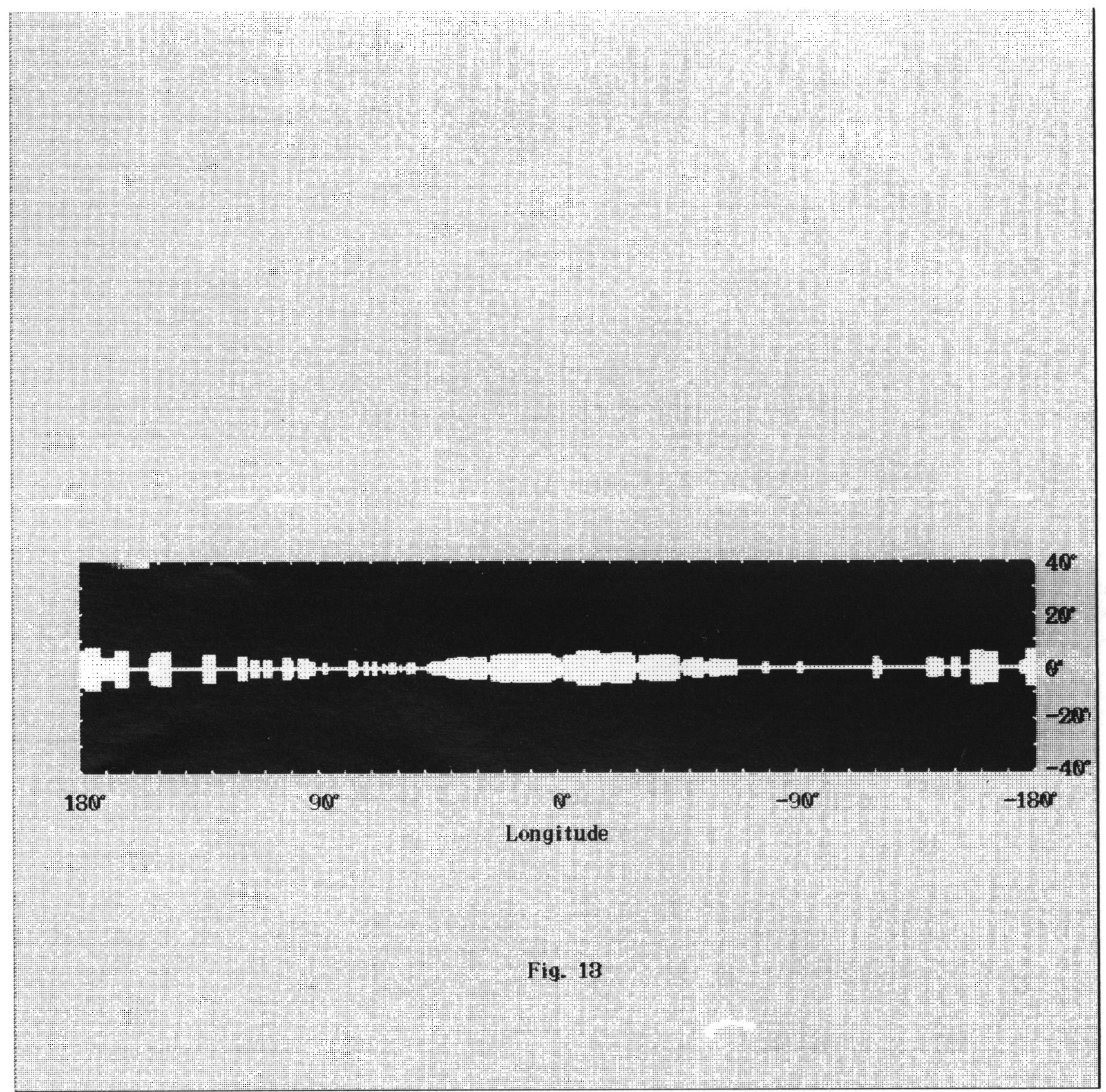

FIG. 13.-Same as Fig. 4 but for exponential disk situation with two-arm spiral pattern

Li AND IKEUCHI (see 73, 409) 


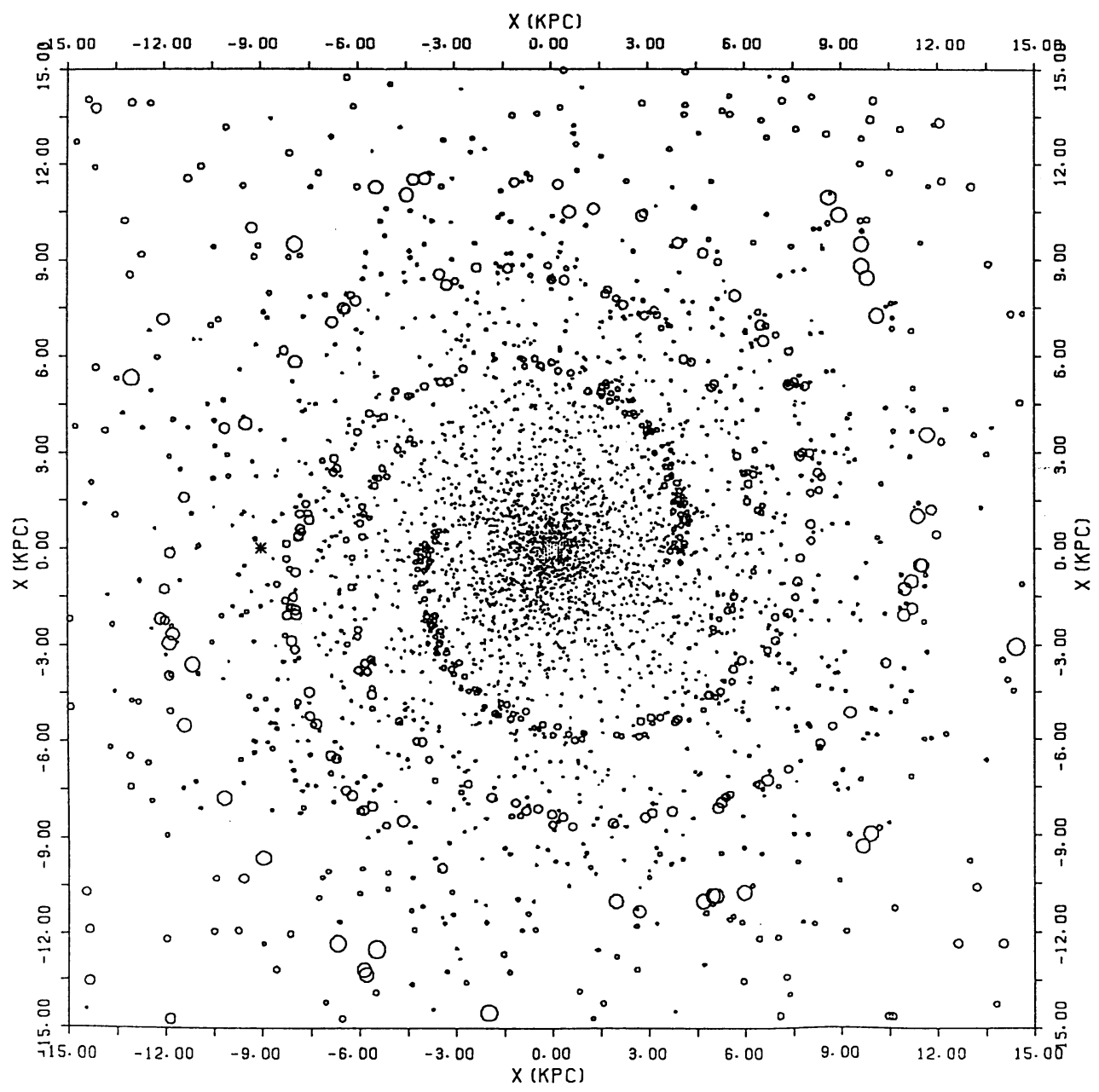

FIG. $11 b$

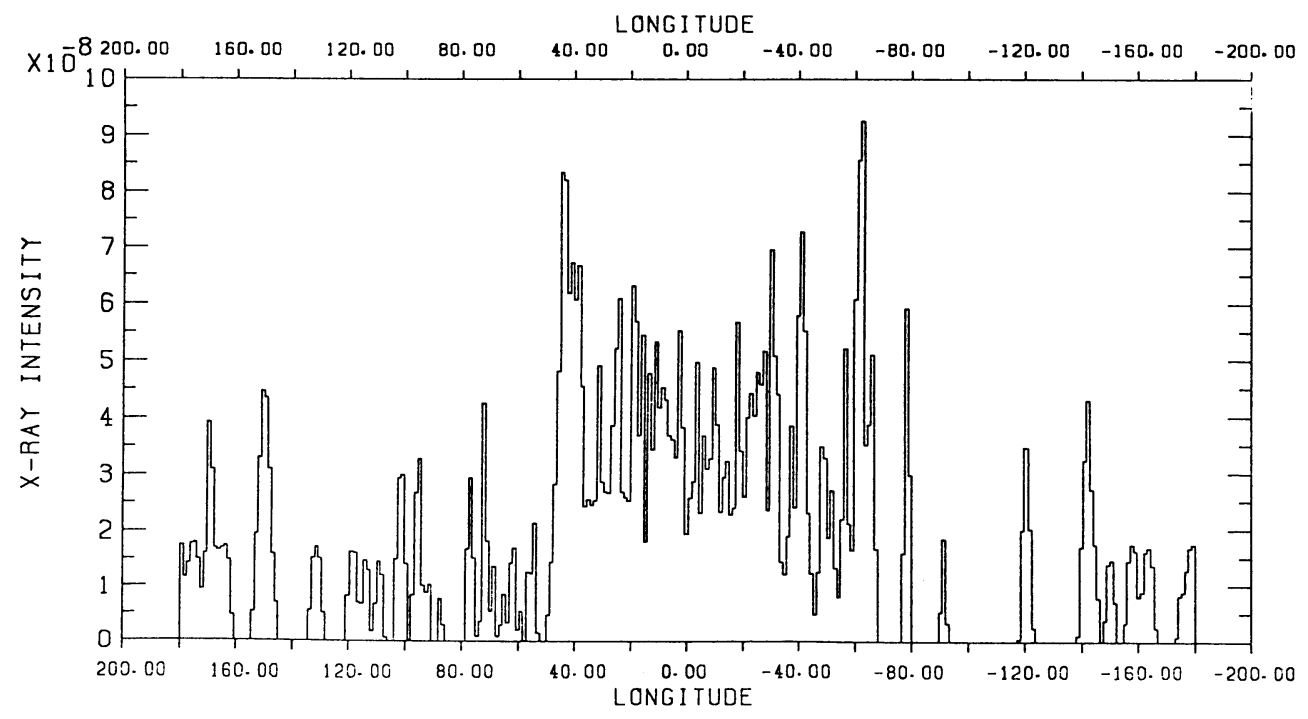

FIG. 14.-Same as Fig. 9 but for exponential disk situation with two-arm spiral pattern 
we observe them from the outside of the spiral galaxy. It is indicated by these observations that the superbubble phenomenon might be very common in spiral galaxies. Here we try to reproduce the distribution of these $\mathrm{H}_{\mathrm{I}}$ holes in M31 as follows.

The observed distribution of dark clouds, $\mathrm{H}$ i regions, and OB associations in the disk of M31 shows they are prominently distributed in a ring in the range $8 \leq R \leq 15 \mathrm{kpc}$ (Nakai and Sofue 1982), while the detailed spiral structure is not well determined. Here we assume an exponential disk takes the form

$$
n \propto e^{-R / 3.5(\mathrm{kpc})} e^{-|z| / 250(\mathrm{pc})},
$$

at $R \geq 8 \mathrm{kpc}$, and the $\mathrm{SBs}$ are formed in proportional to this density. Then we use the exponential Monte Carlo simulation to determine both $R$ and $Z$ distributions of explosion sites of SBs. For illustrating the $\mathrm{H}_{\mathrm{I}}$ holes, we take only those SBs which have broken through the disk since we are seeing from outside. The result is shown in Figure 15 which is very similar to the observed distribution and sizes (Brinks and Bajaja 1986). In this figure, we take account of the inclination of M31 disk, $i=74^{\circ}$, with respect to the line of sight. Therefore, the holes look elliptic as is observed. Since we assume all SBs have circular cross sections along the disk plane, the ellipticities are same for all holes according to the inclination of M31. However, the observed $\mathrm{H}$ I holes show various ellipticities, which imply that the SBs incline to the disk. Further, this deformation will come from the Galactic differential rotation on the expanding shells as is shown by Tenorio-Tagle and Palous (1987). Recently, Palous, Franco, and Tenorio-Tagle (1989) testified that if we consider the effects of the flat rotation curve on the expanding supershells, the observed ellipticities and orientations of $\mathrm{H}$ i holes in M31 and M33 can be interpreted satisfactorily. This is in further support of the chimney model.

It is interesting to study the $\mathrm{H}$ r holes in M31 and M33 in detail, including the comparison of the statistics of the model holes and the observed holes. We will do these in a succeeding paper.

\section{e) Chimneys of the Edge-on Galaxies}

By using the same method used in M31, we try to get the chimneys in an edge-on galaxy like NGC 3079. Only those which are higher than $1 \mathrm{kpc}$ are taken into account. The result is shown in Figure 16 (Plate 24). The result shows that if there are about 1000 superbubbles in the edge-on galaxy, only a dozen or so which are higher than $1 \mathrm{kpc}$ can be expected to be observed.

\section{SUMMARY}

Giving the distribution of SBs and SNRs by the Monte Carlo method under the assumption that they are randomly distributed in the spiral arms and on the Galactic plane, respectively, we study the observational characteristics of the chimney model by H I $21 \mathrm{~cm}$ and X-ray observations. Furthermore, the exponential disk situation described by equation (4) is also calculated. Our results indicate that many $\mathrm{H}_{\mathrm{I}}$ walls stand perpendicular to the Galactic plane which are similar to what have been observed and called supershells by Heiles (1984). They are concentrated in certain longitude regions in the velocity-divided $\mathrm{H}$ I maps which correspond to the accumulation of SBs in spiral arms and concentrated in certain ranges of galactocentric directions in the velocityintegrated maps which imply the compactness of the spiral arms in the inner Galactic region. The number of supershells, their widths and heights, and detectable limiting longitude at a fixed central velocity $V_{0}$ give the information on the spiral structure such as the number of arms and winding manner.

The integrated X-ray emission from SBs and SNRs is also calculated. The distribution of X-ray emissivity shows a ridgelike structure, which is both concentrated in the galactocentric direction and confined to the Galactic disk. Especially in the exponential disk situation, the distribution of $\mathrm{X}$-ray intensity along different directions is relatively smooth which corresponds well to the observed ridge by Warwick et al. (1985) and Koyama et al. (1986), though our model shows a wider extension of the ridge. Since the extension of the ridge of X-ray emissivity depends upon the number and winding manner of arms, more spatially resolved observa-

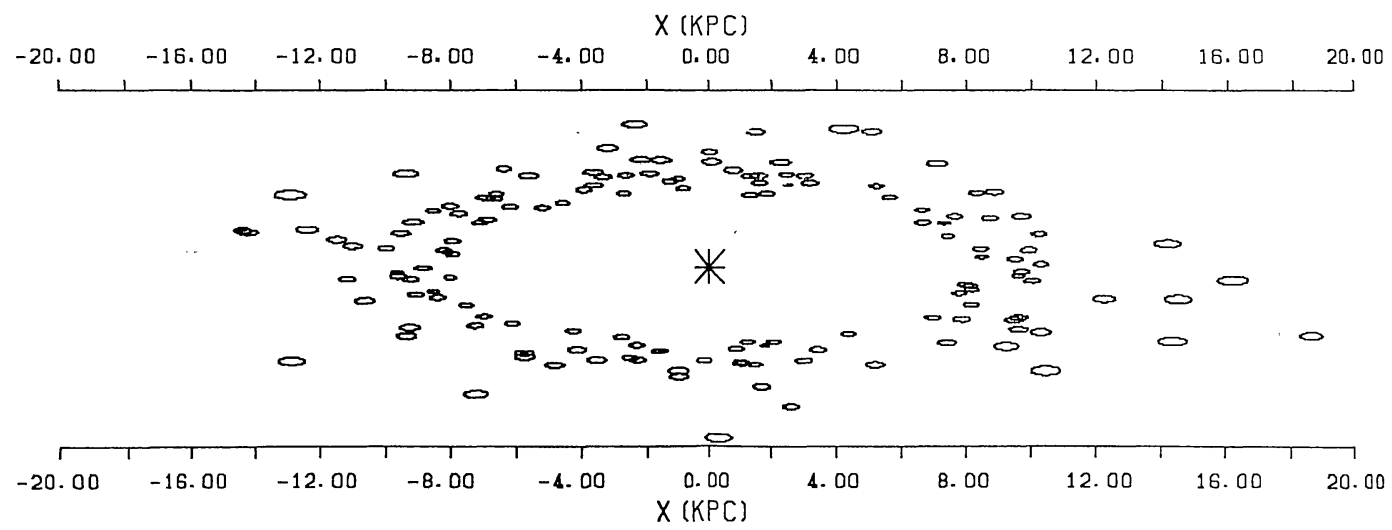

FIG. 15.-Simulated $\mathrm{H}$ I hole distribution in M31 


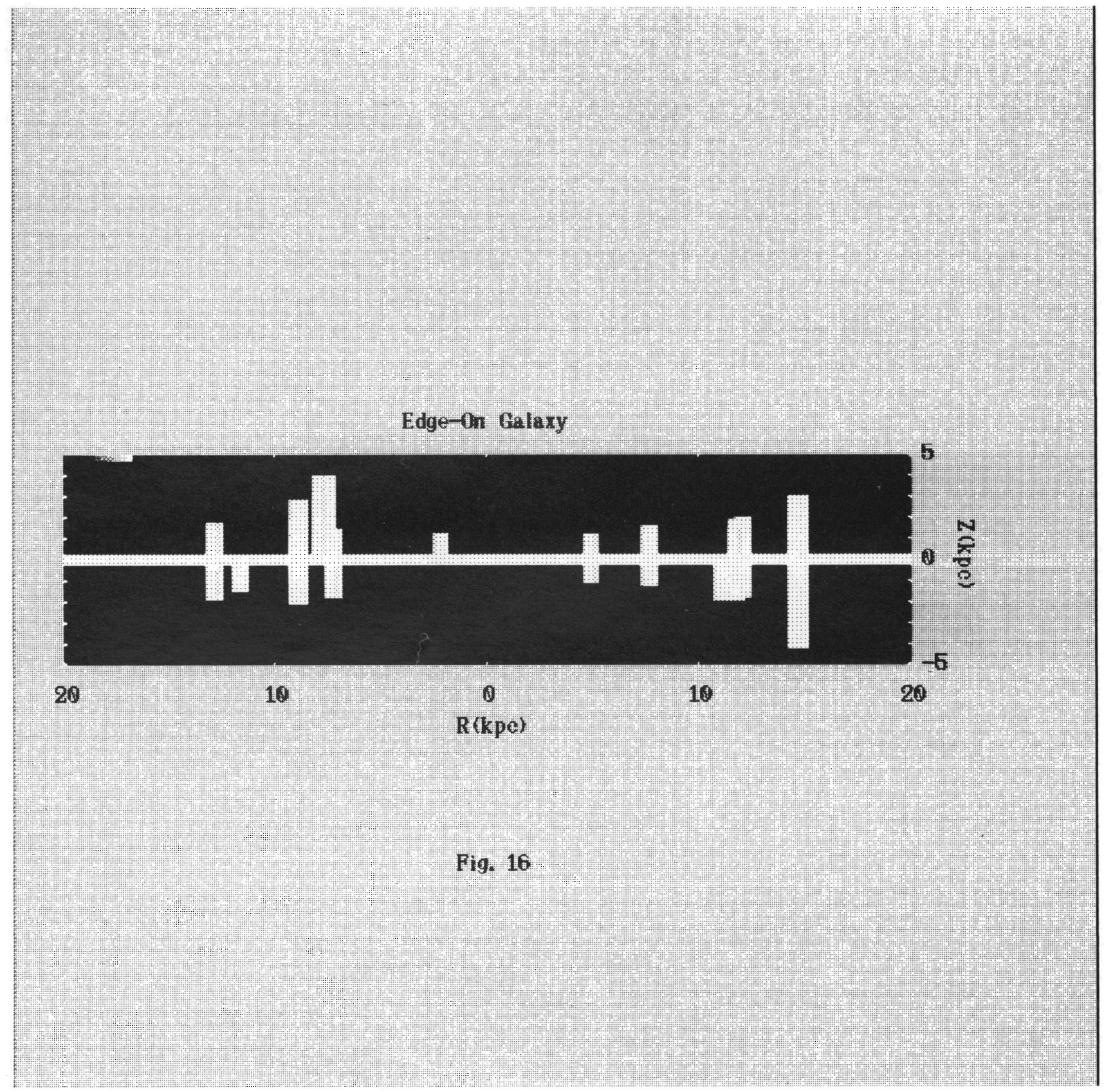

FIG. 16.-Chimneys higher than $1 \mathrm{kpc}$ seen from the outside of an edge-on spiral galaxy like NGC 3079

Li AND IKeuchI (see 73, 411) 
tions are necessary in order to determine them definitely. But the fundamental features support our picture based upon the chimney model.

We further calculate the distribution of $\mathrm{H}$ I holes in inclinating face-on galaxy like M31 seen from outside by assuming an exponential disk. The result corresponds well to the observed $\mathrm{H}$ I holes in M31.

The situation of an edge-on spiral galaxy is also calculated, and giant standing $\mathrm{H}_{\mathrm{I}}$ structures which correspond to the cold walls of the chimneys are expected to be observed in edge-on galaxies.

The authors gratefully acknowledge the colleagues of the theoretical division of National Astronomical Observatory for many valuable discussions. One of the authors (F. L.) acknowledges the scholarships from the Ministry of Education, Science, and Culture of Japan for the graduate studies of foreign students.

\section{REFERENCES}

Audouze, D, and Israel, J 1988, The Cambridge Atlas of Astronomy (Cambridge: Cambridge University Press), p. 308.

Brinks, E., and Bajaja, E. 1986, Astr. Ap., 169, 14.

Bruhweiler, F. C. Gull, T. R., Kafatos, M., and Sofia, S. 1980, Ap. J. (Letters), 238, L27.

Cowie, L. L., Songaila, A., and York, D. 1979, Ap. J, 230, 469

Deul, E., and Hartog, R. H. 1990, Astr. Ap., 229, 362.

Deul, E., and van der Hulst, J. M. 1986, Astr. Ap. Suppl., 67, 509.

Elmegreen, D. M. 1985, in LAU Symposium 106. The Milky Way Galaxy, ed. H. V. Woerden, R. J. Allen, and W. B. Burton (Dordrecht: Reidel), p. 255.

Heiles, C. 1979, $A p . J ., 229,533$. 1984, Ap. J. Suppl., 55, 585

Ikeuchi, S. 1987, in Starbursts and Galaxy Evolution, ed. T. X. Thuan, T. Montmerle, and J. Tran Thanh Van (Paris: Editions Frontières), p. 27. 1988, Fund. Cosmic Phys., 12, 255.

Koyama, K. 1989, Pub. Astr. Soc. Japan, 41, 665.
Koyama, K., Ikeuchi, S., and Tomisaka, K. 1986, Pub. Astr. Soc. Japan, 38, 503 .

Koyama, K., Makishima, K., Tanaka, Y., and Tsunemi, H. 1986, Pub. Astr. Soc. Japan, 38, 121

Li, F. and Ikeuchi, S. 1989, Pub. Astr. Soc. Japan, 4l, 221 (Paper I).

McKee, C. F., and Ostriker, J. P. 1977, Ap. J., 218, 148.

Mihalas, D., and Binney, J. 1981, Galactic Astronomy (San Francisco: W. H. Freeman and Company), p. 529

Nakai, N., and Sofue, Y. 1982, Pub. Astr. Soc. Japan, 34, 561

Norman, C. A., and Ikeuchi, S. 1989, Ap. J., 345, 372.

Palous, J., Franco, J., and Tenorio-Tagle, G. 1989, Astr. Ap., in press.

Raymond, J. C., Cox, D. P., and Smith, B. W. 1976, Ap. J., 204, 290.

Tammann, G. A. 1977, Ann NY Acad. Sci., 302, 61.

Tenorio-Tagle, G., and Palous, J. 1987, Astr. Ap., 186, 287.

Tomisaka, K., Habe, A., and Ikeuchi, S. 1981, Ap. Space Sci., 78, 273.

Tomisaka, K., and Ikeuchi, S. 1986, Pub. Astr. Soc. Japan, 38, 697.

Warwick, R. S., Turner, M. J. L., Watson, M. G., and Willingale, R. 1985, Nature, 317, 218.

Satoru Ikeuchi and Fan Li: Division of Theoretical Astrophysics, National Astronomical Observatory, Mitaka, Tokyo 181, Japan 